\title{
Shape optimization of an acoustic horn
}

\author{
Erik Bängtsson, Daniel Noreland, and Martin Berggren* \\ Department of Information Technology \\ Uppsala University \\ P.O. Box 120 \\ SE-75104 Uppsala, Sweden
}

May 8, 2002

\begin{abstract}
Shape optimization of an acoustic horn is performed with the goal to minimize the portion of the wave that is reflected. The analysis of the acoustical properties of the horn is performed using a finite element method for the Helmholtz equation.

The optimization is performed employing a BFGS Quasi-Newton algorithm, where the gradients are provided by solving the associated adjoint equations. To avoid local solutions to the optimization problem corresponding to irregular shapes of the horn, a filtering technique is used that applies smoothing to the design updates and the gradient. This smoothing technique can be combined with Tikhonov regularization. However, experiments indicate that regularization is redundant for the optimization problems we consider here. However, the use of smoothing is crucial to obtain sensible solutions. The smoothing technique we use is equivalent to choosing a representation of the gradient of the objective function in an inner product involving second derivatives along the design boundary.

Optimization is performed for a number of single frequencies as well as for a band of frequencies. For single frequency optimization, the method shows particularly fast convergence with indications of super-linear convergence close to optimum. For optimization on a range of frequencies, a design was achieved providing a low and even reflection throughout the entire frequency band of interest.
\end{abstract}

Keywords: Shape optimization, Helmholtz equation, finite element method, adjoint equations, smoothing, Tikhonov regularization, BFGS QuasiNewton method, acoustic waveguide, acoustic horn. 


\section{Contents}

1 Introduction 3

2 Problem 5

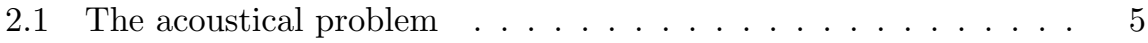

2.2 The optimization problem . . . . . . . . . . . . 7

2.3 The gradient $\nabla_{\alpha} J \ldots \ldots \ldots$. . . . . . . . . . . 8

3 Admissible designs, regularization, smoothing $\quad 10$

3.1 Regularization . . . . . . . . . . . . . . . . . . 10

3.2 Smoothing. . . . . . . . . . . . . . . . 11

4 Discretization $\quad \mathbf{1 4}$

4.1 State equation and objective function . . . . . . . . . . . . 14

4.2 Discrete gradient of $J_{h} \ldots \ldots \ldots \ldots$. . . . . . . . . . . . . . . . . . . . . . . . . 15

4.3 Mesh movement . . . . . . . . . . . . . . . . . . 17

4.4 Discrete regularization and smoothing . . . . . . . . . . . . . 19

4.5 Gradient assembly . . . . . . . . . . . . . . . . 20

4.6 Implementation . . . . . . . . . . . . . . . . . 20

5 Numerical experiments 21

5.1 Experimental setup . . . . . . . . . . . . . . . . . 21

5.2 Single frequency optimization . . . . . . . . . . . . . . 22

5.3 Multi frequency optimization . . . . . . . . . . . . . . . 26

5.3 .1 Two frequencies . . . . . . . . . . . . 26

5.3.2 Three frequencies . . . . . . . . . . . . . . . . . 27

5.3.3 Many frequencies ................ . . . . 27

5.4 Mesh dependency . . . . . . . . . . . . . . . . . . . . . 33

6 Conclusions $\quad 35$

7 Acknowledgments $\quad 36$

A Gradient derivation $\quad 39$

A.1 Preliminaries . . . . . . . . . . . . . . . . . 39

A.2 Differentiation of $J \ldots \ldots \ldots . \ldots \ldots 40 . \ldots \ldots$

A.3 The gradient $\nabla_{\alpha} J \ldots \ldots \ldots 42$

A.4 Derivation of $\nabla_{\eta} J^{\epsilon} \ldots \ldots \ldots \ldots$. . . . . . . . . . . . . . . . . . . . . . . .

B Gradient derivation in the discrete case $\quad \mathbf{4 5}$

B.1 Preliminaries . . . . . . . . . . . . . . . . 45

B.2 Derivation ..................... 46 


\section{Introduction}

The problem of analyzing the impedance and radiation properties of acoustic horns has been treated extensively $[1,3,4,9,14]$. The methods employed range from one-dimensional transmission line models to two- or three-dimensional finite element and boundary element methods, all of which are accurate within their own realms of validity. However, surprisingly little has been reported in the literature about the problem of designing a horn with a prescribed set of properties.

The purpose of this article is to present a fast and accurate method by which it is possible to design a horn that provides an impedance matching between a waveguide and the surrounding air within a given design frequency range. The method is based on an accurate mathematical model in two space dimensions that is capable of accurately describing the radiation impedance of the horn as well as effects associated to modal conversion. Although the target impedance curve of the horns is real and constant in the experiments presented here, our method is applicable also for other impedance curves, after minor alterations. The computational complexity of the method increases with the number of frequencies considered since the analysis is performed in the frequency domain. However, the method is fast in the sense that the computational complexity is essentially independent of the number of design variables.

If a design is known whose properties are good but not ideal, it is usually sufficient to gradually modify the geometry until a desired set of properties is reached, a procedure usually denoted shape optimization. Here, we formulate the shape optimization problem as a multipoint nonlinear optimization problem that is solved using a quasi-Newton algorithm. This is a well-know approach in other disciplines, used to optimize geometries for structural [19] or aerodynamic [12] performance, for instance.

To the best of our knowledge, there have been no reported results in the literature from using such an approach for the design of acoustic horns. However, there are certainly other related works regarding acoustic shape optimization, for instance by Christensen and Olhoff [5] and Habbal [11]. Christensen and Olhoff [5] optimize the shape of a loudspeaker with respect to its directivity. A boundary integral method is employed to solve the sound propagation problem, and the optimization is performed using a sequential linear programming algorithm with the objective-function gradient approximated by finite differences. Habbal [11] considers shape optimization of a sound barrier using a finite element formulation of the acoustic problem. The shape optimization problem is solved using a gradient based search method where the gradient is provided by solving the associated adjoint equations. For completeness, the work by Kausel [16] and Amir et al. [2] should also be mentioned, although the underlying physical models in both these papers are one-dimensional transmission line approximations. Kausel [16] uses a zeroth-order gradient free search method in order to optimize the impedance spectrum of a brass instrument. Amir et al. [2] propose a method for bore reconstruction based on pulse reflectometry.

Among non-acoustic applications, of interest is the work done by Lee et al. [17] and Ratnajeevan et al. [21], who consider optimization of waveguides for microwaves. In these papers, the steepest-descent algorithm is employed with gradients provided from the adjoint method or by finite differences.

The rest of the article is organized as follows. Section 2 introduces the acous- 
tical problem, contains a short derivation of the mathematical model we use, and presents the optimization problem together with the method for finding the gradient from solving the associated adjoint equations. The importance of using some strategy to force or at least promote smooth design became apparent for us during the course of this work. The approaches we advocate, regularization and smoothing, are presented in section 3. Section 4 is devoted to the finite element discretization of the problem, as well as a description of the mesh movement strategy. The numerical experiments we performed are reported in section 5, and the main portion of the text ends with a discussion and some conclusions in section 6 . The appendix contains detailed derivations of different expressions that are used throughout the article. 


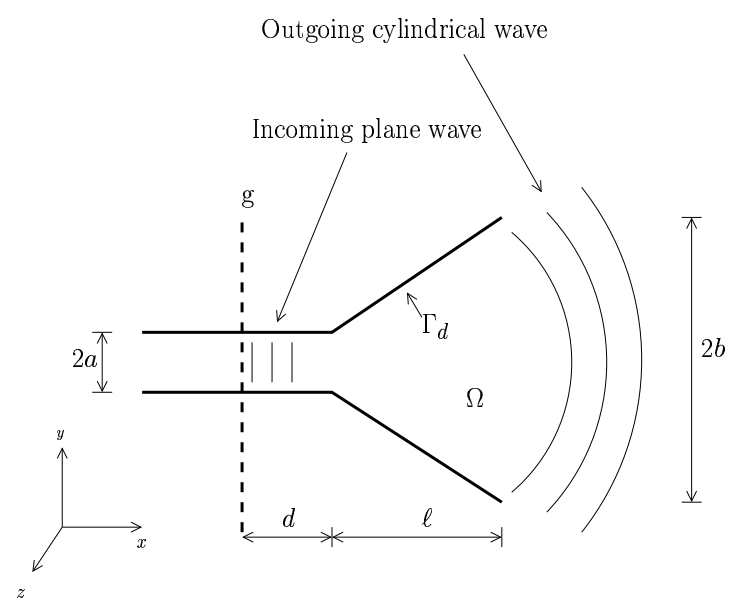

Figure 1: The acoustical horn to be optimized. An infinite planar wave guide is connected to the horn.

\section{Problem}

\subsection{The acoustical problem}

Throughout the following, it is assumed that wave propagation is governed by the linear wave equation for the acoustical pressure $P$, that is

$$
\frac{\partial^{2} P}{\partial t^{2}}=c^{2} \Delta P
$$

in a lossless adiabatic medium. Here, $c$ is the speed of sound, and $\Delta$ denotes the Laplacian operator. The geometry of interest is shown in figure 1. It consists of a planar channel, the waveguide, with infinite extension to the left and with width $2 a$. The conical termination of the waveguide will be denoted the horn, and its initial shape is a conical hopper with width $2 a$ at the connection to the waveguide and width $2 b$ in the free end. For simplicity, the geometry is considered infinite in the direction normal to the plane, which effectively reduces the problem to two space dimensions.

A wave propagating in a waveguide can be expressed as a superposition of modal components satisfying the wave equation and the boundary conditions. It is known from wave physics that in a waveguide, modes whose frequency is below the cut-off frequency are evanescent along the waveguide. With the source of the wave incident in the horn placed far away to the left, only planar modes are fed into the horn if the source frequency is lower than the cut-off frequency of all non-planar modes. Furthermore, if the distance $d$ is large enough to ensure that the non-planar modes of the wave reflected in the horn are extinct, the wave propagating over the cross section $g$ is a superposition of two planar waves.

Due to the choice of numerical method to solve the wave propagation problem, it has to be modeled on a bounded region, like $\Omega$ in figure 2 . On the cross section $g$ in figure 1 , the inflow boundary $\Gamma_{\text {in }}$ is placed, and $\Gamma_{\text {out }}$ is placed in the far-field zone of the horn.

As the wave propagation problem in this case by assumption is infinite and non-echoic, suitable artificial boundary conditions have to be imposed on $\Gamma_{i n}$ 


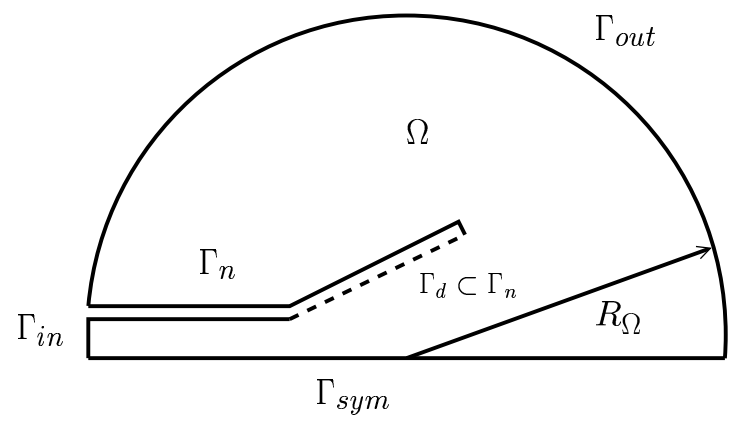

Figure 2: The modeled situation. Note that the design boundary $\Gamma_{d}$ is a part of $\Gamma_{n}$.

and $\Gamma_{\text {out }}$. On $\Gamma_{\text {in }}$, the amplitude of an incoming planar wave, the incident wave, should be specified, whereas the outgoing planar wave, the reflected wave, should be left unaffected. Let $\boldsymbol{x}$ denote spatial points and $\boldsymbol{n}$ the outward-directed unit normal on the boundary of $\Omega$. Assuming that the normal on $\Gamma_{i n}$ points along the waveguide, single-frequency, planar waves in the waveguide can be written as

$$
P(\boldsymbol{x}, t)=A e^{i(k \boldsymbol{x} \cdot \boldsymbol{n}+\omega t)}+B e^{i(-k \boldsymbol{x} \cdot \boldsymbol{n}+\omega t)} \quad A, B \in \mathbb{C},
$$

where $k=\omega / c$ is the wavenumber, and $\omega$ is the angular frequency of the wave. The first and second terms in (2) correspond to the incident and reflected waves, respectively. Differentiating expression $(2)$ at $\Gamma_{i n}$ yields

$$
\begin{aligned}
& \frac{\partial P}{\partial t}=A i \omega e^{i(k \boldsymbol{x} \cdot \boldsymbol{n}+\omega t)}+B i \omega e^{i(-k \boldsymbol{x} \cdot \boldsymbol{n}+\omega t)} \\
& \frac{\partial P}{\partial n}=A i k e^{i(k \boldsymbol{x} \cdot \boldsymbol{n}+\omega t)}-B i k e^{i(-k \boldsymbol{x} \cdot \boldsymbol{n}+\omega t)}
\end{aligned}
$$

where $\partial P / \partial n$ denotes derivative in the outward-directed normal direction. Combining expressions (3) and (4) and using $k c=\omega$ we find that

$$
\frac{\partial P}{\partial t}+c \frac{\partial P}{\partial n}=2 i \omega A e^{i(k \boldsymbol{x} \cdot \boldsymbol{n}+\omega t)} .
$$

Condition (5) is satisfied for each wave of the type (2) and can thus be used to set the amplitude $A$ of the incident wave without affecting the amplitude $B$ of the reflected wave.

An appropriate absorbing boundary condition in the farfield $\Gamma_{\text {out }}$ is the firstorder radiation condition in cylindrical coordinates [8],

$$
\frac{\partial P}{\partial t}+c \frac{\partial P}{\partial n}+\frac{c}{2 R_{\Omega}} P=0
$$

This will cause no reflection at $\Gamma_{\text {out }}$ of waves propagating strictly in the normal direction.

On the remaining boundaries, $\Gamma_{n}$, assumed to consist of sound hard material, and $\Gamma_{\text {sym }}$, a symmetry plane, the appropriate condition

$$
\frac{\partial P}{\partial n}=0 \quad \text { on } \Gamma_{n} \cup \Gamma_{\text {sym }}
$$


is imposed.

The mathematical model of the acoustical problem described above consists of equation (1) together with boundary conditions (5), (6), and (7). Making use of the ansatz $P(\boldsymbol{x}, t)=p(\boldsymbol{x}) e^{i \omega t}$ for single-frequency, time harmonic solutions, we obtain the following Helmholtz equation for the complex amplitude function $p$

$$
\begin{aligned}
c^{2} \Delta p+\omega^{2} p & =0 & & \text { in } \Omega, \\
\left(i \omega+\frac{c}{2 R_{\Omega}}\right) p+c \frac{\partial p}{\partial n} & =0 & & \text { on } \Gamma_{\text {out }}, \\
i \omega p+c \frac{\partial p}{\partial n} & =2 i \omega A & & \text { on } \Gamma_{\text {in }}, \\
\frac{\partial p}{\partial n} & =0 & & \text { on } \Gamma_{n} \cup \Gamma_{\text {sym }} .
\end{aligned}
$$

With a terminology borrowed from systems theory, we denote equation (8) the state equation.

\subsection{The optimization problem}

The primary interest of the shape optimization is to minimize the reflection in the horn. This corresponds to matching the impedance of the horn to the waveguide and the surrounding air. The reflection coefficient $R$ is defined as the quotient between the amplitude of the reflected wave and the amplitude of the incident wave. ${ }^{1}$ From this definition, it follows that for a time-harmonic wave like (2), $R$ in the waveguide is the magnitude of the second term divided with the magnitude of the first term, that is

$$
R=\frac{B}{A}
$$

The reflected wave is observed on the inflow boundary $\Gamma_{i n}$. If the origin of the coordinate system is located on $\Gamma_{i n}$, the amplitude $p$ at this point can be written as $p=A+B$ according to (2). The magnitude of the reflected wave on the inflow boundary is thus given by $B=p-A$ and the objective function $J$ is defined as the square of the magnitude of the reflected wave integrated over the inflow boundary, that is

$$
J=\frac{1}{2} \int_{\Gamma_{\text {in }}}|p-A|^{2} d \Gamma .
$$

If the pressure is constant over $\Gamma_{i n}$ (as is assumed in expression (2)), then

$$
J=a|R A|^{2} .
$$

The value of the objective function depends on the design of the horn, that is, on the shape of $\Gamma_{d}$. In order to define a set of admissible designs, we first define the reference boundary $\Gamma_{d}^{r e f}$ as the straight line connecting the start and end points of the design boundary (figure 3). The admissible designs will be

\footnotetext{
${ }^{1} \mathrm{~A}$ generalized reflection coefficient including also higher order modes can be defined, but in this work only planar modes are prevalent, by construction.
} 


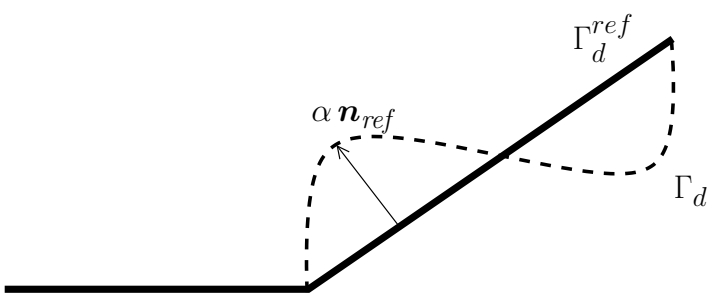

Figure 3: The design boundary

chosen among normal deflections from the reference design. That is, each point $\boldsymbol{x}$ on an admissible boundary $\Gamma_{d}$ is associated with some $\boldsymbol{x}_{r e f} \in \Gamma_{d}^{r e f}$ through

$$
\boldsymbol{x}=\boldsymbol{x}_{r e f}+\alpha\left(\boldsymbol{x}_{r e f}\right) \boldsymbol{n}_{r e f},
$$

where $\boldsymbol{n}_{\text {ref }}$ is the unit normal on $\Gamma_{d}^{r e f}$ and $\alpha \in U_{\text {ad }}$, where $U_{\text {ad }}$ is a convex subset of smooth, real-valued function on $\Gamma_{d}^{r e f}$ vanishing at the end points. The choice of $U_{\mathrm{ad}}$ is further discussed in $\S 3$.

Hence, the optimization problem will be:

$$
\begin{gathered}
\text { Find } \alpha_{*} \in U_{\text {ad }} \text { such that } \\
J\left(\alpha_{*}\right) \leq J(\alpha) \quad \forall \alpha \in U_{\text {ad }} .
\end{gathered}
$$

To find the displacement $\alpha$, we will use a standard, well-tested gradient-based optimization algorithm: a quasi-Newton method with BFGS secant approximations of the Hessian together with a globalization strategy provided by cubic back-tracking. Further details on the method can be found in [7].

The computation of the gradient of the objective function must be inexpensive and exact in order to obtain an efficient optimization: inexpensive since the operation is done (at least) once per iteration and exact to obtain a good Hessian approximation and fast convergence of the optimization.

\subsection{The gradient $\nabla_{\alpha} J$}

Consider a given design $\Gamma_{d}$ associated with some function $\alpha$. The objective function depends on $\alpha$ only through $p$. This we denote

$$
J(\alpha)=\frac{1}{2} \int_{\Gamma_{i n}}|p(\alpha)-A|^{2} d \Gamma
$$

Let $\delta \alpha=\alpha^{\prime}-\alpha$ be a variation of $\alpha$ (that is, a difference of two admissible designs $\alpha$ and $\alpha^{\prime}$ ). The differential of $J$ at the current design is

$$
\delta J(\alpha)=\lim _{s \rightarrow 0} \frac{J(\alpha+s \delta \alpha)-J(\alpha)}{s}
$$

If $J$ is differentiable, the differential is given by a linear functional, the gradient (or the Frechet derivative), $\nabla_{\alpha} J$ operating on the variation $\delta \alpha$. We use the notation

$$
\delta J=\left\langle\nabla_{\alpha} J, \delta \alpha\right\rangle
$$


As an example, if $\alpha$ is finite dimensional and spanned by $N$ basis functions $w_{k}(x)$, then, for each $x \in \Gamma_{d}^{r e f}$,

$$
\alpha(\boldsymbol{x})=\sum_{k=1}^{N} \alpha_{k} w_{k}(\boldsymbol{x}) \quad \alpha_{k} \in \mathbb{R},
$$

and the components of the gradient are given by

$$
\begin{aligned}
\frac{\partial J}{\partial \alpha_{k}}=\lim _{s \rightarrow 0} \frac{J\left(\alpha+s w_{k}\right)-J(\alpha)}{s} & =\left\langle\nabla_{\alpha} J, w_{k}\right\rangle \\
\forall k & =1, \ldots, N
\end{aligned}
$$

The partial derivatives (17) can be approximated by finite differences,

$$
\frac{\partial J}{\partial \alpha_{k}} \approx \frac{J\left(\alpha+\epsilon w_{k}\right)-J(\alpha)}{\epsilon},
$$

where $\epsilon>0$ is a chosen step length. This is a simple and nonintrusive way to calculate gradients, as no knowledge of the specifics of the state equation discretization is needed. However, the computational cost increases linearly with the number of design variables $N$, becoming prohibitively expensive when $N$ is large, particularly for fine meshes. Furthermore, the accuracy of the gradient is strongly dependent on the choice of step length.

Far more efficient and exact is the adjoint equation approach. The procedure and the final expressions are given here. For the derivation, we refer to Appendix A.

1. For a given $\alpha$, solve state equation (8) with $\Gamma_{d}$ given by $\alpha$.

2. Given the pressure distribution $p$ from step 1 solve the adjoint equation

$$
\begin{aligned}
c^{2} \Delta z+\omega^{2} z & =0 & & \text { in } \Omega, \\
\left(-i \omega+\frac{c}{2 R}\right) z+c \frac{\partial z}{\partial n} & =0 & & \text { on } \Gamma_{\text {out }}, \\
-i \omega z+c \frac{\partial z}{\partial n} & =p-A & & \text { on } \Gamma_{\text {in }}, \\
\frac{\partial z}{\partial n} & =0 & & \text { on } \Gamma_{n} \cup \Gamma_{d} \cup \Gamma_{\text {sym }} .
\end{aligned}
$$

3. The gradient is given by

$$
\nabla_{\alpha} J=\gamma \mathfrak{R e}\left(\omega^{2} \bar{z}_{\alpha} p_{\alpha}-c^{2} \nabla \bar{z}_{\alpha} \cdot \nabla p_{\alpha}\right),
$$

where $p_{\alpha}$ and $z_{\alpha}$ are observations of the state and adjoint equations along $\Gamma_{d}$, and where $\gamma$ is a metric term; for precise definitions, see Appendix A.3.

Note that the computation of the gradient requires one state solve and one adjoint solve regardless of the dimension of $\alpha$.

If $\alpha$ is given by (16), then

$$
\frac{\partial J}{\partial \alpha_{k}}=\int_{\Gamma_{d}^{r e f}} w_{k} \nabla_{\alpha} J d \Gamma, \quad k=1, \ldots, N .
$$




\section{Admissible designs, regularization, smoothing}

The three-step procedure in $\S 2.3$ yields expression (20) for the gradient of the objective function (10). A discrete version of this procedure, as presented in $\S 4$ below, is all that is needed to apply a gradient-based optimization algorithm such as the steepest-descent, conjugate gradient, or a quasi-Newton method with secant approximations of the model Hessian. However, such a straightforward approach is not likely to be successful for reasons outlined below. In $\S 3.1$ and $\S 3.2$ we present strategies to address these problems. The discussion is restricted to the "continuous" case, before applying discretization. Corresponding expression in the discrete case are given in $\S 4.4$ below.

There are at least three issues that can cause problems with a naive implementation of a gradient-based shape optimization:

(i) Existence of solutions. It is necessary to request some smoothness on the candidate functions $\alpha$ in order to obtain a manufacturable design. However, defining $U_{\text {ad }}$ to be, say, the space of continuously differentiable functions vanishing at the end point, there is no guarantee that problem (12) has any solutions. This is a well-known complication associated with shape optimization problem associated with elliptic boundary-value problems, such as the Helmholtz equation. The problem is that the sensitivity of the objective function to oscillatory changes in the design may be low, which may cause a minimizing sequence of the objective function to produce increasingly oscillatory shapes. In general, conditions to suppress oscillations of the design boundary have to provided. See [20] for further details.

(ii) Intermediate, wiggly shapes. Even if there indeed exists a smooth solution to the optimization problem without imposing any strong constraints on the admissible designs, intermediate shapes produced by the optimization algorithm in the search for an optimal solution, may be wiggly enough to cause problems for the mesh movement algorithm.

(iii) Local minima. There are reasons to expect the existence of local minima of the objective function associated with wiggly shapes, particularly at the end points of the design boundary, and the optimization algorithm could be trapped in such a local minimum.

\subsection{Regularization}

The current optimization problem is conceptually a nonlinear least-squares problem. A common cure to irregular solutions, as well as a means to provide existence of solutions to nonlinear least-squares problem is Tikhonov regularization [23]. A reasonable choice of regularization in the present context is to bound the integral-square of the second derivatives. This quantity is roughly the curvature of the design boundary for small $\alpha$. Such a regularization can be obtained in the following manner.

Introducing a new design variable $\eta: \Gamma_{d}^{r e f} \rightarrow \mathbb{R}$, we compute the function $\alpha$ defining the design boundary by solving the Poisson problem

$$
\begin{aligned}
-\alpha^{\prime \prime} & =\eta & & \text { on } \Gamma_{d}^{r e f}, \\
\alpha & =0 & & \text { at the end points of } \Gamma_{d}^{r e f} .
\end{aligned}
$$


The regularized objective function will then be

$$
J^{\epsilon}(\eta)=\frac{\epsilon}{2} \int_{\Gamma_{d}^{r e f}} \eta^{2} d \Gamma+\frac{1}{2} \int_{\Gamma_{i n}}|p(\eta)-A|^{2} d \Gamma,
$$

and the shape optimization problem becomes:

Find $\eta_{*} \in U_{\text {ad }}$ such that

$$
J^{\epsilon}\left(\eta_{*}\right) \leq J^{\epsilon}(\eta) \quad \forall \eta \in U_{\text {ad }},
$$

where $U_{\text {ad }}$ now can be chosen simply as the space of square-integrable functions on $\Gamma_{d}^{r e f}, L^{2}\left(\Gamma_{d}^{r e f}\right)$. The use of regularization of this sort is not new in shape optimization. It has been used in the context of aerodynamic shape optimization by Gunzburger et al. [10] and Mohammadi and Pironneu [18], for instance.

Assume that $\eta_{*}$ minimizes $J^{\epsilon}$. The first term of the objective function guarantees that $\eta_{*}=\alpha_{*}^{\prime \prime}$ will be square integrable as long as $\epsilon>0$. By the so-called Sobolev inequalities, see $[6, \mathrm{IV}, \S 8]$, this means that $\alpha_{*}$ will be at least a continuously differentiable function. Thus, the regularization prevents excessive oscillations as long as $\epsilon>0$. Requesting $\alpha$ to satisfy equation (22) also imposes the constraint that $\alpha$ should vanish at the end points.

The design variable is now $\eta$ instead of $\alpha$. We thus need an expression for the gradient of $J^{\epsilon}$ with respect to $\eta$. The following procedure, derived in Appendix A.4 provides this.

1. For a given design variable $\eta$, solve equation (22) for $\alpha$.

2. Given $\alpha$, solve state equation (8) with the design boundary $\Gamma_{d}$ defined by $\alpha$ through equation (11).

3. Given the pressure field from step 2, solve the adjoint equation (19) for $z$.

4. From the solution $p$ to the state equation, the solution $z$ of the adjoint equation, compute the gradient $\nabla_{\alpha} J$ given by expression (20).

5. Given $\nabla_{\alpha} J$, solve the Poisson problem

$$
\begin{aligned}
-r^{\prime \prime} & =\nabla_{\alpha} J & & \text { on } \Gamma_{d}^{r e f}, \\
r & =0 & & \text { at the end points of } \Gamma_{d}^{r e f} .
\end{aligned}
$$

6. The gradient of $J^{\epsilon}$ with respect to $\eta$ is then given by

$$
\nabla_{\eta} J^{\epsilon}=\epsilon \eta+r .
$$

Steps 2,3 , and 4 above are exactly the same as in the unregularized case in $\S 2.3$. The difference is in steps 1 and 5, where a "filtering" or "smoothing" is applied to the design variable $\eta$ and the gradient $\nabla_{\alpha} J$, respectively.

\subsection{Smoothing}

Now assume that the solution to the unregularized problem (12) is smooth, without the active enforcement of bounds on oscillations in $\alpha$. To our surprise, 
numerical experiments strongly suggest that this indeed is the case for the frequency range of interest, that is, when the horn is efficient as an impedance transformer. Tikhonov regularization is not desirable in this case since the solution will then be suboptimal with respect to the unregularized problem. Furthermore, the unregularized problem formulation is also problematic with respect to the risk for intermediate wiggly shapes and local minima.

In this case we still advocate the use of the regularized formulation, but with $\epsilon=0$. We use the term smoothing for this approach; it is motivated by steps 1 and 5 in the procedure of $\S 3.1$. Note that problems (12) and (24) with $\epsilon=0$ are equivalent for smooth solutions. To see this, consider the condition for optimality of problem (12), $\nabla_{\alpha} J=0$. This may correspond to a global as well as a local minimum. Since equation (25) has a unique solution, corresponding $\nabla_{\alpha} J$ vanishes. Since $\epsilon=0$, we see from expression (26) that also $\nabla_{\eta} J^{\epsilon}$ vanishes. Likewise, if $\nabla_{\eta} J^{\epsilon}=0$ for $\epsilon=0$, equation (25) also yields that $\nabla_{\alpha} J=0$. Thus, the use of smoothing does not change the solutions of the optimization problem: it only constitutes a change of variables.

However, the paths in design space generated by the iterations in the optimization algorithm will be dramatically different whether or not smoothing is used. A gradient-based optimization algorithm relies on derivative and possibly second-derivative information and is thus by necessity a local approach. Thus, the optimization algorithm will start out with iterates that are close to the reference configuration and will proceed further and further away. What is "local", "close", and "further away" depends on the norm in question. Here is where formulations (12) and (24) with $\epsilon=0$ differ. The norm for formulation (12) is

$$
\|\alpha\|_{0}^{2}=\int_{\Gamma_{d}^{r e f}} \alpha^{2} d \Gamma,
$$

that is, the $L^{2}\left(\Gamma_{d}^{r e f}\right)$ norm, whereas, by equation (22), the implied norm for the $\alpha$ 's in formulation (24), is

$$
\|\alpha\|_{2}^{2}=\int_{\Gamma_{d}^{r e f}} \eta^{2} d \Gamma=\int_{\Gamma_{d}^{r e f}}\left(\alpha^{\prime \prime}\right)^{2} d \Gamma,
$$

which defines a norm in $H_{0}^{2}\left(\Gamma_{d}^{r e f}\right)^{2}$. For an highly oscillatory function $\alpha_{o s c}$ with a small amplitude we have that $\left\|\alpha_{\text {osc }}\right\|_{0} \ll\left\|\alpha_{\text {osc }}\right\|_{2}$.

"Local" and "close" for formulation (12), associated with the norm (27) include highly oscillatory, discontinuous, and even singular functions $\alpha$. In contrast, local deviations from the reference configuration with respect to the norm (28) only contains continuously differentiable functions $\alpha$. Hence, the use of smoothing will produce a preference for smooth design updates, and we can expect the optimization algorithm to generate very smooth shapes at the first iterates, and that additional details to the shape are added as the iterations proceed. This cannot be expected when not using smoothing. Thus, the use of smoothing should be an efficient cure to problems (ii) and (iii) above: intermediate, wiggly shapes, and local minima associated with wiggly shapes. This of course assumes that there is a smooth solution to the unregularized problem. However, if the optimization problem happen to have a desired solution that

$$
{ }^{2} H_{0}^{2}\left(\Gamma_{d}^{r e f}\right)=\left\{\alpha \mid \alpha, \alpha^{\prime}, \alpha^{\prime \prime} \in L^{2}\left(\Gamma_{d}^{r e f}\right), \alpha=\alpha^{\prime}=0 \text { at the end points of } \Gamma_{d}^{r e f}\right\}
$$


is not smooth, containing corners for instance, it may take many iterations to resolve the optimal geometry when using smoothing.

Remark 3.1. The reasoning above applies to the chosen BFGS-Quasi-Newton algorithm as well as the steepest-descent and non-linear conjugate-gradient method. However, Newton's method with full Hessian information, a method we do not use, would act differently. The Newton step is independent of the choice of norm in which the gradient and Hessian are represented. However, the quadratic model assumed in Newton's method is valid only locally. To account for this, all useful implementations of Newton's method involve a globalization strategy. One example is the trust-region approach, in which the quadratic model is trusted only in a neighborhood around the current iterate. See [7] for further details. Here is where the choice of norm becomes crucial, and we expect the stronger norm associated with the smoothing to be advantageous when solving the trust-region subproblem. We believe that the trust-region approach would be a better choice for a full-fledged Newton algorithm than the line-search strategy that we use here, since the trust-region approach utilize the norm more intrusively to modify the step.

Remark 3.2. Gradient smoothing, that is, some version of equation (25) is advocated by Jameson et al. [13] as a means to provide smoother design updates. We use the same term, "smoothing", for our approach, although it is slightly different. Conceptually, the difference is that we prefer to view the smoothing as a change of variables, or rather a change of inner product in which the gradient is represented. In practice, the difference manifests itself in that our approach leads to a smoothing for the design updates in addition to smoothing of the gradient. 


\section{Discretization}

There are two conceptually different approaches to discretize the above optimization problem. The most obvious one is to choose suitable discretizations to state equation (8), adjoint equation (19) and gradient expression (21). This is sometimes labeled "differentiate-then-discretize" or the "continuous approach". Finding a suitable discretization of the adjoint equation is straightforward due to its close resemblance to the state equation. However, gradient expression (21) contains evaluation of gradients along $\Gamma_{d}$ and a metric term, and finding an accurate discretization of this is far from trivial. Unfortunately, high accuracy of the gradient directions is crucial to get good convergence of the optimization algorithm, in particular since we use gradients to construct Hessian approximations.

A more robust approach, and the one that we use, is the "discrete approach", or "discretize-then-differentiate". In this case, the state equation and the objective function are discretized and from those expressions the associated, discrete adjoint equation and corresponding gradient expression are derived. This yields, in exact arithmetics, an exact expression for the gradient with respect to the discretized objective function and, within the limits of the chosen algorithm, the best possible convergence of the optimization algorithm regardless of the resolution.

\subsection{State equation and objective function}

The domain $\Omega$ is triangulated using elements $\left\{T_{m}\right\}_{m=1}^{M}$, and the union of the triangles forms $\Omega_{h}$ approximating $\Omega$. In the triangle vertices, a set of node points, $\left\{\boldsymbol{x}_{k}\right\}_{k \in \mathcal{K}}$ is defined, where $\mathcal{K}$ is the index set of node numbers.

On $\Omega_{h}$, the solution to the acoustic problem $p$ is approximated in the space $V_{h}$ of continuous, complex-valued functions being linear on each triangle. We have

$$
V_{h}=\operatorname{span}\left\{w_{j}\right\}_{j \in \mathcal{K}} \subset H^{1}\left(\Omega_{h}\right),
$$

where $w_{j}$ are standard nodal basis functions such that

$$
w_{j}\left(\boldsymbol{x}_{k}\right)= \begin{cases}1 & \text { if } k=j, \\ 0 & \text { if } k \neq j\end{cases}
$$

and $H^{1}\left(\Omega_{h}\right)$ is the space of complex-valued functions on $\Omega_{h}$ such that the function itself and both partial derivatives are square integrable.

The finite-element approximation to the solution $p$ of state equation (8) is denoted $p_{h}$. The function $p_{h}$, a linear combination of the basis functions $w_{j}$,

$$
p_{h}(\boldsymbol{x})=\sum_{j \in \mathcal{K}} p_{j} w_{j}(\boldsymbol{x}), \quad p_{j} \in \mathbb{C},
$$


is the solution to equation ${ }^{3}$

$$
\begin{aligned}
& c^{2} \int_{\Omega_{h}} \nabla w_{j} \cdot \nabla p_{h} d \Omega-\omega^{2} \int_{\Omega_{h}} w_{j} p_{h} d \Omega+i \omega c \int_{\Gamma_{\text {in }} \cup \Gamma_{\text {out }}} w_{j} p_{h} d \Gamma+ \\
& \frac{c^{2}}{2 R_{\Omega}} \int_{\Gamma_{\text {out }}} w_{j} p_{h} d \Gamma=2 i \omega c A \int_{\Gamma_{\text {in }}} w_{j} d \Gamma \quad \forall j \in \mathcal{K},
\end{aligned}
$$

or in matrix form

$$
\left(c^{2} \mathbf{K}-\omega^{2} \mathbf{M}+i \omega c \mathbf{M}^{\text {in }, \text { out }}+\frac{c^{2}}{2 R_{\Omega}} \mathbf{M}^{\text {out }}\right) \mathbf{p}=2 i \omega c A \mathbf{M}^{\text {in }} \mathbf{e},
$$

where $\mathbf{p}=\left(p_{1}, p_{2}, \ldots\right)^{T}$ and $\mathbf{e}=(1, \ldots, 1)^{T}$. The stiffness and mass matrices $\mathbf{K}$ and $\mathbf{M}$ have components $K_{j k}=\int_{\Omega_{h}} \nabla w_{j} \cdot \nabla w_{k} d \Omega$ and $M_{j k}=\int_{\Omega_{h}} w_{j} w_{k} d \Omega$. Moreover, $\mathbf{M}^{\text {in }}$ denotes the matrix with components $M_{j k}^{\mathrm{in}}=\int_{\Gamma_{i n}} w_{j} w_{k} d \Gamma$. Analogous definitions hold for $\mathbf{M}^{\text {out }}$ and $\mathbf{M}^{\text {in,out }}$.

Objective function (10) is discretized by substituting $p$ with $p_{h}$,

$$
J_{h}=\frac{1}{2} \int_{\Gamma_{\text {in }}}\left|p_{h}-A\right|^{2} d \Gamma,
$$

or in matrix form,

$$
J_{h}=\frac{1}{2}(\mathbf{p}-A \mathbf{e})^{H} \mathbf{M}^{\mathrm{in}}(\mathbf{p}-A \mathbf{e})
$$

where superscript $H$ denotes the combination of complex conjugate and transposition.

\subsection{Discrete gradient of $J_{h}$}

Let $\boldsymbol{x}_{k}, k \in \mathcal{K}$, be an arbitrary node point in the mesh. We will derive an expression for the partial derivative of objective function (32) with respect to a variation $\delta \boldsymbol{x}_{k}$ of mesh point $\boldsymbol{x}_{k}$. Section 4.5 below will describe how to use this information to construct gradients with respect to the design variables. Only a formal derivation in matrix notation is presented here. The actual derivation is made with the equations in variational form. This conceptually similar but more tedious calculation can be found in Appendix B. For guidance, references to corresponding expression in Appendix B are given throughout this section.

Equation (31) can be written symbolically as

$$
\mathbf{L}\left(\mathbf{p}, \boldsymbol{x}_{k}\right)=0
$$

Differentiating with respect to a variation $\delta \boldsymbol{x}_{k}$, we obtain

$$
\mathbf{L}_{\mathbf{p}} \delta \boldsymbol{p}+\mathbf{L}_{\boldsymbol{x}_{k}} \delta \boldsymbol{x}_{k}=0,
$$

the sum of two discrete differentials of the state equation, where the matrices $\mathbf{L}_{\mathbf{p}}$ and $\mathbf{L}_{\boldsymbol{x}_{k}}$ are derivatives of the state equation with respect to the pressure and node point $\boldsymbol{x}_{k}$ respectively. (This step corresponds to expression (B.12).)

\footnotetext{
${ }^{3}$ ignoring the fact that integrals over $\Gamma_{\text {out }}$ should in reality be over a piecewise-linear approximation $\Gamma_{\text {out }}^{h}$ of $\Gamma_{\text {out }}$.
} 
The differential of objective function (33) is

$$
\delta J=\mathfrak{R e}\left[\delta \mathbf{p}^{H} \mathbf{M}^{\mathrm{in}}(\boldsymbol{p}-A \mathbf{e})\right],
$$

(which corresponds to expression (B.7)). Taking the complex conjugate of equation (34) and multiplying it from the right with an arbitrary vector $\mathbf{z}$ yields

$$
\delta \mathbf{p}^{H} \mathbf{L}_{\mathbf{p}}^{H} \mathbf{z}+\delta \boldsymbol{x}_{k}^{H} \mathbf{L}_{\boldsymbol{x}_{k}}^{H} \mathbf{z}=0,
$$

(which corresponds to expression (B.15)).

So far $\mathbf{z}$ has been an arbitrary vector. Now chose $\mathbf{z}$ to be the solution of the adjoint equation,

$$
\mathbf{L}_{\mathbf{p}}^{H} \mathbf{z}=\mathbf{M}^{\mathrm{in}}(\mathbf{p}-A \mathbf{e}),
$$

(which corresponds to equation (B.16) or equation (41) below). Substituting equation (37) into equation (36) yields

$$
\delta \mathbf{p}^{H} \mathbf{M}^{\text {in }}(\mathbf{p}-A \mathbf{e})+\delta \boldsymbol{x}_{k}^{H} \mathbf{L}_{\boldsymbol{x}_{k}}^{H} \mathbf{z}=0
$$

(corresponding to expression (B.18)).

Expression (38) can now be used to eliminate $\delta \mathbf{p}$ from expression (35), which becomes

$$
\delta J_{h}=\mathfrak{R e}\left(-\delta \boldsymbol{x}_{k}^{H} \mathbf{L}_{\boldsymbol{x}_{k}}^{H} \mathbf{z}\right)=\delta \boldsymbol{x}_{k}^{T}\left[\mathfrak{R e}\left(-\mathbf{L}_{\boldsymbol{x}_{k}}^{H} \mathbf{z}\right)\right],
$$

where we use that $\delta \boldsymbol{x}_{k}$ is real in the second equality. This implies that

$$
\frac{\partial J_{h}}{\partial \boldsymbol{x}_{k}}=-\mathfrak{R e}\left(\mathbf{L}_{\boldsymbol{x}_{k}}^{H} \mathbf{z}\right) .
$$

When this derivation is performed explicitly, we show in Appendix B that

$$
\begin{aligned}
\frac{\partial J_{h}}{\partial \boldsymbol{x}_{k}} & =\mathfrak{R e}\left\{c^{2} \int_{\Omega_{h}} z_{h}\left(\nabla w_{k} \cdot \nabla \bar{p}_{h}\right)+c^{2} \int_{\Omega_{h}} \nabla \bar{p}_{h}\left(\nabla z_{h} \cdot \nabla w_{k}\right)\right. \\
& \left.-c^{2} \int_{\Omega_{h}} \nabla w_{k}\left(\nabla z_{h} \cdot \nabla \bar{p}_{h}\right)+\omega^{2} \int_{\Omega_{h}} \nabla w_{k}\left(z_{h} \bar{p}_{h}\right)\right\},
\end{aligned}
$$

where $p_{h}$ is the solution to state equation (30) and $z_{h} \in V_{h}$ is the solution to the following discrete adjoint equation:

$$
\begin{aligned}
c^{2} \int_{\Omega} \nabla w_{j} & \cdot \nabla z_{h} d \Omega-\omega^{2} \int_{\Omega} w_{j} z_{h} d \Omega-i \omega c \int_{\Gamma_{\text {in }} \cup \Gamma_{\text {out }}} w_{j} z_{h} d \Gamma \\
+ & \frac{c^{2}}{2 R_{\Omega}} \int_{\Gamma_{\text {out }}} w_{j} z_{h} d \Gamma=\int_{\Gamma_{\text {in }}} w_{j}\left(p_{h}-A\right) d \Gamma \quad \forall j \in K,
\end{aligned}
$$

or, in matrix form,

$$
\left(c^{2} \mathbf{K}-\omega^{2} \mathbf{M}-i \omega c \mathbf{M}^{\mathrm{in}, \text { out }}+\frac{c^{2}}{2 R_{\Omega}} \mathbf{M}^{\text {out }}\right) \mathbf{z}=\mathbf{M}^{\mathrm{in}}(\mathbf{p}-A \mathbf{e}),
$$

where $\mathbf{z}$ contains the coefficients in an expansion of $z_{h}$ in the nodal basis for $V_{h}$, similarly as for $p_{h}$ in expression (29). 


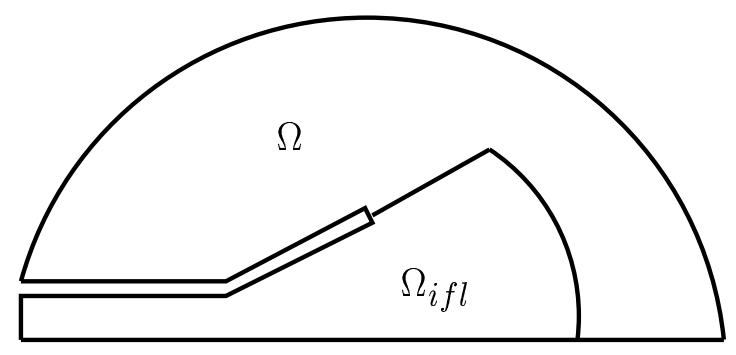

FiguRE 4: The problem geometry with the influence region $\Omega_{i f l}$ denoted

\subsection{Mesh movement}

During the optimization process, the design boundary is displaced from $\Gamma_{d}^{r e f}$ to an updated position, described by the design parameter $\alpha$. For each new, improved design it is necessary to solve the state equation in order to compute the corresponding objective function.

As the design boundary moves, it is necessary to change the mesh, also in the interior of $\Omega_{h}$, to retain mesh quality. This can be done in various ways, and one option is to regenerate the mesh for each new $\Gamma_{d}$. Unfortunately, this will introduce noise in the objective function, since the generation of a new mesh will add and delete mesh nodes in an unpredictable manner. Such noise may be detrimental to the convergence of the optimization algorithm.

We have chosen to generate an initial mesh that is successively modified during the entire optimization by displacements of nodes in the interior of $\Omega_{h}$. Thus, the value of the objective function will depend on each point in the mesh that is allowed to move; the dependency will of course be weak for mesh nodes far from $\Gamma_{d}$. The mesh movement strategy must gradually and smoothly distribute the design changes into the mesh in order to prevent a noisy objective function response to design variation.

Such a mesh movement strategy can be realized in different ways. One way is to employ a model where the displacement depends on some function, for instance exponential or quadratic, of the distance to the design boundary. This is complicated and expensive to implement for unstructured meshes, since the distance to the design boundary for each interior node must be computed at each optimization step. Another model for the mesh point updates is to use a spring analogy where each node is treated as a point mass connected to its neighbor nodes with springs. When the design boundary is moved, a displacement field is induced in the mesh via the springs (typically a linearized model for small displacements is used).

We have chosen a slightly more elaborate strategy, treating the domain $\Omega$ as a linearly elastic solid. This introduces tangential stiffness with respect to local normal displacements, which should improve mesh quality. We consider an initial guess of the design boundary $\Gamma_{d}^{i n i t}$ and the associated deflection $\alpha^{\text {init }}$ with respect to the normal direction $\boldsymbol{n}_{\text {ref }}$ of the reference boundary $\Gamma_{d}^{r e f}$ (figure 5). The domain $\Omega$ associated with $\Gamma_{d}^{i n i t}$ is then triangulated, which yields a list of mesh nodes $\mathbf{x}^{\text {init }}=\left(\boldsymbol{x}_{1}^{\text {init }}, \boldsymbol{x}_{2}^{\text {init }}, \ldots\right)^{T}$. When we need to consider a particular design, associated with some function $\alpha$, each node point in the mesh is displaced according to $\boldsymbol{x}_{k}=\boldsymbol{x}_{k}^{\text {init }}+\delta \boldsymbol{x}_{k}$, where $\delta \boldsymbol{x}_{k}$ is obtained as a numerical evaluation, 


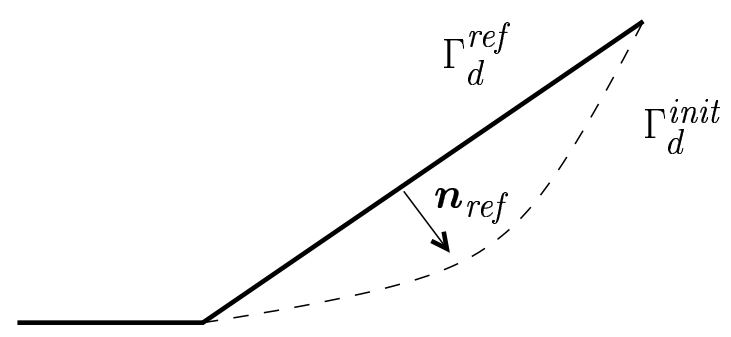

Figure 5: The relation between $\Gamma_{d}^{\text {init }}$ and $\Gamma_{d}^{\text {ref }}$.

at mesh node $\boldsymbol{x}_{k}$, of the solution $\boldsymbol{u}$ to Navier's equation for an inhomogeneous solid,

$$
\begin{array}{ll}
\nabla\left(\frac{\nu E(\boldsymbol{x})}{1-2 \nu} \nabla \cdot \boldsymbol{u}\right)+\nabla \cdot\left\{\frac{E(\boldsymbol{x})}{2}\left[\nabla \boldsymbol{u}+(\nabla \boldsymbol{u})^{T}\right]\right\}=\mathbf{0} & \text { in } \Omega, \\
\boldsymbol{u}=\left(\alpha-\alpha^{i n i t}\right) \boldsymbol{n}_{r e f} & \text { on } \Gamma_{d}^{i n i t}, \\
\boldsymbol{u}=\mathbf{0} & \text { on } \partial \Omega \backslash \Gamma^{i n}
\end{array}
$$

The coefficients $E(\boldsymbol{x})$ and $\nu$ are the Youngs modulus and the Poissons ratio, material parameters describing the mechanical properties of the solid. The reason to use a model with an inhomogeneous Youngs modulus is explained below. The equations (43) are discretized using the same finite element method as the state and adjoint equation.

Solving equation (43) provides a mapping from $\left(\alpha-\alpha^{i n i t}\right) \boldsymbol{n}_{r e f}$, the deflection of the current design $\Gamma_{d}$ from the initial design $\Gamma_{d}^{i n i t}$, to deflections in the interior, $\boldsymbol{u}$. After discretization, solving the discrete counterpart to equation (43) provides a mapping from $\delta \boldsymbol{\alpha}$, the discrete version of $\left(\alpha-\alpha^{i n i t}\right) \boldsymbol{n}_{r e f}$, to $\delta \mathbf{x}=\left(\delta \boldsymbol{x}_{1}, \delta \boldsymbol{x}_{2}, \ldots\right)$, which approximates $\boldsymbol{u}$ at the mesh nodes. This mapping is linear, and can thus be written

$$
\delta \mathbf{x}=\mathbf{A} \delta \boldsymbol{\alpha} .
$$

The dimension of the vector $\delta \boldsymbol{\alpha}$ is $N$, the number of node points at the design boundary. Each element of $\delta \boldsymbol{\alpha}$ yields the deflection, in the direction of $\boldsymbol{n}_{\text {ref }}$, needed to move corresponding mesh point from $\Gamma_{d}^{i n i t}$ to $\Gamma_{d}$. The dimension of $\delta \boldsymbol{x}$ is equal to the number of mesh points in $\Omega$.

We have chosen to compute the matrix $\mathbf{A}$, column by column, in a preprocessing step. This is done by numerically solving equation (43) $N$ times, for unit displacements of each node on the design boundary. This is advantageous when $N$ is not too large. An alternative would be to compute the action of $\mathbf{A}$, by solving the discrete version of equation (43) after each update of the design boundary.

If $n$, the number of mesh nodes, is large, the computation of $\mathbf{A}$ is expensive since equation (43) has to be solved once for each design node. To make the computation cheaper, only a fraction of $\Omega$, called the influence region and denoted $\Omega_{i f l}$ in figure 4 , is regarded as a linearly elastic solid. $\Omega_{i f l}$ is chosen as the $k$ mesh nodes most affected by design deflections.

The linear assumption in (43) is reasonable only if the displacement $\alpha-\alpha^{\text {init }}$ is sufficiently small. Otherwise the mesh topology may be corrupted. To avoid 
this, two strategies are implemented. The first one is to choose the initial guess of design boundary, $\Gamma_{d}^{\text {init }}$, as a shape closer to the optimal one than $\Gamma_{d}^{\text {ref }}$. If it is chosen sufficiently close, the design displacement required to achieve an optimal shape of the horn is small enough to satisfy the linear assumption in (43).

The second strategy is to transfer large mesh displacements to regions with large elements where they are better absorbed, something that is obtained if the solid is stiffer in regions with small elements. This inhomogeneous stiffness is controlled via the Youngs modulus. The solid is made stiffer in regions with small elements by letting $E$ be inversely proportional to the area of the triangles in the region, that is

$$
E(\boldsymbol{x}) \propto \frac{1}{\left|T_{n}\right|} \quad \boldsymbol{x} \in T_{n},
$$

where $T_{n}$ is the $n$th triangular element and $\left|T_{n}\right|$ is the area of it relative the area of the largest triangle. Note that the inhomogeneous Youngs modulus is evaluated on the initial mesh and no updates of its value is performed during the optimization. Hence, as the elements are suppressed by the mesh movements, they will not become stiffer and stiffer.

\subsection{Discrete regularization and smoothing}

On $\Gamma_{d}^{r e f}$, the design variable $\eta$ and the deflection $\alpha$ are approximated as a linear combination of standard FEM 1D nodal basis functions $w_{j}(\boldsymbol{x}), \boldsymbol{x} \in \Gamma_{d}^{r e f}$, such that

$$
\eta_{h}=\sum_{j=1}^{N} \eta_{j} w_{j}(\boldsymbol{x}), \quad \alpha_{h}=\sum_{j=1}^{N} \alpha_{j} w_{j}(\boldsymbol{x}) .
$$

The finite-element discretization of the design smoothing (22) is

$$
\int_{\Gamma_{d}^{r e f}} \nabla w_{j} \cdot \nabla \alpha_{h} d \Gamma=\int_{\Gamma_{d}^{r e f}} w_{j} \eta_{h} d \Gamma=0 \quad j=1, \ldots, N,
$$

or in matrix ${ }^{4}$ form

$$
\tilde{\mathbf{K}} \boldsymbol{\alpha}=\tilde{\mathbf{M}} \boldsymbol{\eta},
$$

where $\boldsymbol{\alpha}=\left(\alpha_{1}, \ldots, \alpha_{N}\right)^{T}, \boldsymbol{\eta}=\left(\eta_{1}, \ldots, \eta_{N}\right)^{T}, \tilde{K}_{j k}=\int_{\Gamma_{d}^{r e f}} \nabla w_{j} \cdot \nabla w_{k} d \Gamma$ and $\tilde{M}_{j k}=\int_{\Gamma_{d}^{r e f}} w_{j} w_{k} d \Gamma$.

The discrete smoothed gradient $r_{h}$ can also be expressed in the same basis as above,

$$
r_{h}=\sum_{j=1}^{N} r_{j} w_{j}(\boldsymbol{x}),
$$

and the matrix form of the discrete smoothing of the gradient, corresponding to equation (25), will be

$$
\tilde{\mathbf{K}} \mathbf{r}=\nabla_{\boldsymbol{\alpha}} J_{h},
$$

where

$$
\mathbf{r}=\left(r_{1}, \ldots, r_{N}\right)^{T}, \quad \nabla_{\boldsymbol{\alpha}} J_{h}=\left(\frac{\partial J_{h}}{\partial \alpha_{1}}, \ldots \frac{\partial J_{h}}{\partial \alpha_{N}}\right)^{T} .
$$

\footnotetext{
${ }^{4}$ The "tilde" mark are added on the stiffness and mass matrices to distinguish them from corresponding matrices in expression (31).
} 
Thus the discrete gradient of the regularized objective function with respect to $\boldsymbol{\eta}$ can be expressed

$$
\nabla_{\boldsymbol{\eta}} J_{h}^{\epsilon}=\epsilon \boldsymbol{\eta}+\mathbf{r} .
$$

\subsection{Gradient assembly}

Appendix B (and the formal derivation of $\S 4.2$ ) yields the gradient with respect to each node point in the mesh. In this section we show how the matrix $\mathbf{A}$ used in the mesh movement strategy of $\S 4.3$ can be utilized to obtain an expression for the gradient in terms of the design variables $\boldsymbol{\alpha}$ or $\boldsymbol{\eta}$.

The differential of $J$ is the action of the gradient $\nabla_{\mathbf{x}} J$ with respect to a variation $\delta \mathbf{x}$ of the node points of the mesh,

$$
\delta J=\delta \mathbf{x}^{T} \nabla_{\mathbf{x}} J
$$

From $\S 4.3$, we obtain the relation between $\delta \boldsymbol{\alpha}$ and $\delta \mathbf{x}$. Inserting expression (44) into (50) yields

$$
\delta J=\delta \boldsymbol{\alpha}^{T} \mathbf{A}^{T} \nabla_{\mathbf{x}} J
$$

implying that

$$
\nabla_{\boldsymbol{\alpha}} J=\mathbf{A}^{T} \nabla_{\mathbf{x}} J .
$$

Expressions (48) and (49) finally imply that

$$
\nabla_{\boldsymbol{\eta}} J^{\epsilon}=\epsilon \boldsymbol{\eta}+\tilde{\mathbf{K}}^{-1} \mathbf{A}^{T} \nabla_{\mathbf{x}} J .
$$

\subsection{Implementation}

We use the software Femlab from the Comsol Group for assembling the matrices in state equation (31), adjoint equation (42), the smoothers (47) and (48), and the discrete counterpart to equation (43) involved in the mesh movement. Femlab is also utilized for meshing and postprocessing. The gradient with respect to each node point, expression (40), is computed by a single loop over all triangles. For efficiency, this routine is written in Fortran 90, compiled as a Mex file, which allows the routine to be accessed as a Matlab function.

For optimization, we use a quasi-Newton method with the BFGS update for the model Hessian and cubic polynomial linesearch, as implemented in the function fminunc from Matlab's Optimization Toolbox. The code required to paste together the different routines is written in Matlab. 


\section{$5 \quad$ Numerical experiments}

\section{$5.1 \quad$ Experimental setup}

TABle 1: Geometry data used for the numerical experiments. Parameters defined as in figure 1 and figure 2 .

\begin{tabular}{|c|c|c|c|c|}
\hline$a(\mathrm{~m})$ & $b(\mathrm{~m})$ & $\ell(\mathrm{m})$ & $d(\mathrm{~m})$ & $R_{\Omega}(\mathrm{m})$ \\
\hline \hline 0.05 & 0.3 & 0.5 & 0.5 & 1.0 \\
\hline
\end{tabular}

We will report numerical experiments optimizing the horn in figure 1 . The dimensions of the setup are given in table 1 . These were chosen in order to resemble a reasonably realistic loudspeaker horn. The frequency range of interest for the optimization is given by the physical dimensions of the horn. The lower frequency bound is dictated by the maximum wavelength for which the dimensions of the horn are large enough for it to function as an impedance transformer from the waveguide into free air. The lowest frequency for which it was possible to obtain an impedance match showed to be slightly above $100 \mathrm{~Hz}$. The upper frequency bound, on the other hand, is chosen such that only plane waves are propagating in the planar waveguide attached to the horn, that is, all modes with a $y$-dependence in the waveguide are evanescent. The general solution to the Helmholtz equation in a $2 \mathrm{D}$ waveguide can be written

$$
p(x, y)=\left(A e^{-i \sqrt{\omega^{2} / c^{2}-\zeta^{2}} x}+B e^{i \sqrt{\omega^{2} / c^{2}-\zeta^{2}} x}\right)\left(C e^{-i \zeta y}+D e^{i \zeta y}\right),
$$

where $\zeta=n \pi c /(2 a), n=0,1,2, \ldots$. The cut-off frequency $f_{c}$ of a higher order mode is associated with the longitudinal wavenumber becoming imaginary. This occurs at $\omega_{c}=n \pi c /(2 a)$ or $f_{c}=n c /(4 a)$. With $a=0.05 \mathrm{~m}$ and $c=345 \mathrm{~m} / \mathrm{s}$, we have $f_{c}=1725 \mathrm{~Hz}$ for $n=1$. At $850 \mathrm{~Hz}$, the amplitude of the first non-planar mode will decay with a factor of around $10^{6}$ within a distance of $\ell=0.5 \mathrm{~m}$. Thus, setting the upper frequency bound to $850 \mathrm{~Hz}$, the higher mode contamination at $\Gamma_{i n}$ can thus be expected to be negligible.

TABLE 2: Data for the unstructured finite element meshes where $n$ is the number of nodes, $m$ is the number of elements, $N$ is the number of design variables, and $h_{\max }$ is the length of the longest element edge in the mesh.

\begin{tabular}{|c||c|c|c|c|}
\hline Mesh & $n$ & $m$ & $N$ & $h_{\max }(\mathrm{m})$ \\
\hline \hline I & 662 & 1165 & 14 & 0.1 \\
II & 2488 & 4660 & 29 & 0.05 \\
III & 9635 & 18640 & 59 & 0.025 \\
\hline
\end{tabular}

Three finite element meshes, denoted Mesh I, II and III, with different resolution have been used to triangulate $\Omega$. The three meshes are related in such a way that Mesh II is a uniform refinement of Mesh I and Mesh III is a uniform refinement of Mesh II. The mesh properties are found in table 2 .

A rough estimate of the grid resolution needed to numerically resolve the wavelengths of interest can be found from the following one-dimensional analysis. Consider a grid of constant step size $h$, on which the equation $p_{x x}+k^{2} p=0$ is solved using continuous functions, linear on each element. The numerical 


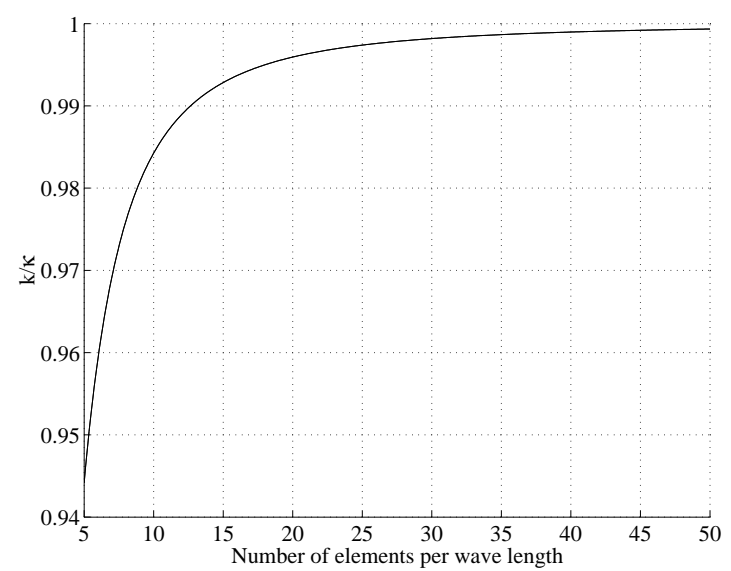

FiguRE 6: The quotient between the wavenumber $k$ and the numerical wavenumber $\kappa$ as a function of the number of elements per wavelength using a simple one-dimensional analysis.

wavenumber $\kappa$ can be shown to satisfy the equation

$$
\cos (\kappa h)=\frac{1-\frac{h^{2} k^{2}}{3}}{1+\frac{h^{2} k^{2}}{6}} .
$$

The quotient between the wavenumber $k$ and the numerical wavenumber $\kappa$ is plotted versus the number of elements per wavelength in figure 6 . A low value of this quotient indicate a high degree of numerical dispersion. It can be seen that the difference between $k$ and $\kappa$ is smaller than $1 \%$ when the the number of elements per wavelength is is larger than 13. Allowing no more than this degree of dispersion and choosing $h=h_{\max }$ for Meshes I, II, and III, the largest allowed frequencies for the three meshes are 288, 575 and $1150 \mathrm{~Hz}$, respectively.

The square of the absolute value of the sound pressure distribution corresponding to the initial shape of the horn is presented in figure 8 . The state equation is solved on Mesh II for $550 \mathrm{~Hz}$, and the dark bands in the waveguide indicate reflections in the horn.

The numerical experiments presented in this section were performed on a SUN Enterprise 420 computer with UltraSPARC-II $450 \mathrm{MHz}$ processors.

\subsection{Single frequency optimization}

As a first test, shape optimization for three single frequencies with and without smoothing was performed on mesh II. For reasons of resolution, we limit the highest frequency to $550 \mathrm{~Hz}$, and the other two frequencies are chosen as 400 $\mathrm{Hz}$ and $280 \mathrm{~Hz}$.

Figure 9 shows the designs obtained for the three frequencies, with and without smoothing. No regularization was employed, that is $\epsilon=0$ (cf. $\S 3$ ). Using smoothing, nice and smooth designs are achieved and the corresponding reflection spectra shown in figure 11 exhibit clear minima for the design frequencies. The square of the absolute value of the sound pressure distribution corresponding to the optimal design for $550 \mathrm{~Hz}$ is shown in figure 12. The dark bands in the 


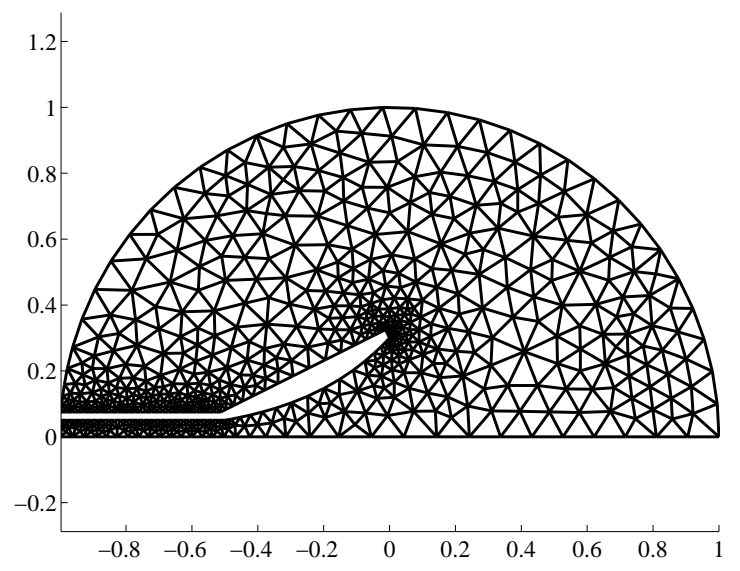

Figure 7: The finite element mesh, denoted Mesh I in table 2, on the initial geometry. Note that the $\Gamma_{d}^{\text {init }}$ is different from $\Gamma_{d}^{\text {ref }}$.

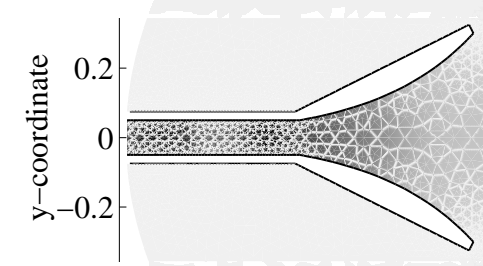

Figure 8: The square of the absolute value of the initial sound pressure in the horn, the waveguide, and the surroundings. Note the banded pattern in the waveguide, indicating reflections.

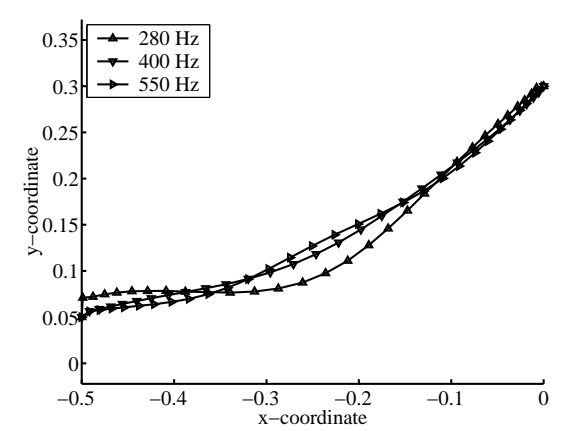

(a) Without smoothing

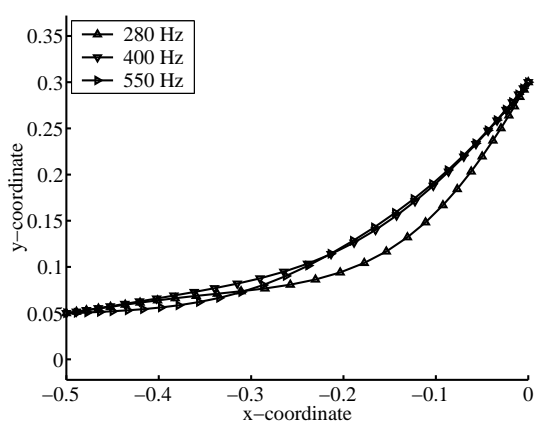

(b) With smoothing

Figure 9: Optimized design boundaries for three single frequencies; $280 \mathrm{~Hz}$, $400 \mathrm{~Hz}$, and $550 \mathrm{~Hz}$. 


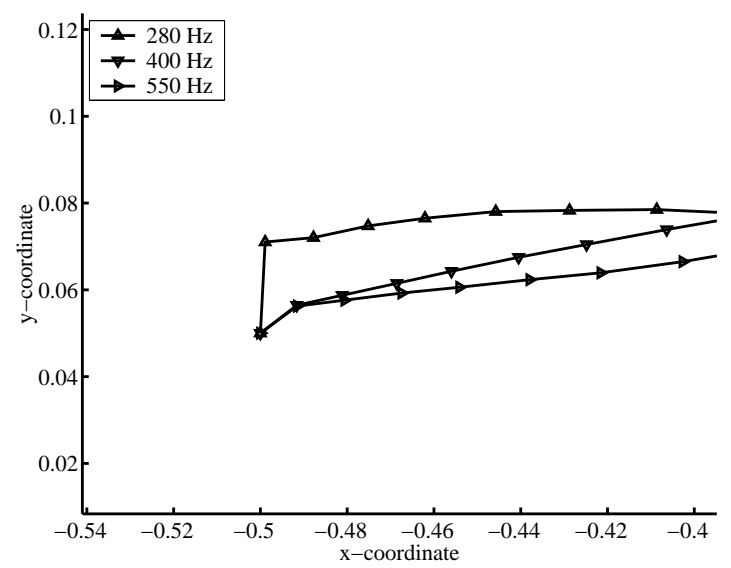

Figure 10: A detail of the optimized design boundary for the three single frequencies $280 \mathrm{~Hz}, 400 \mathrm{~Hz}$, and $550 \mathrm{~Hz}$. Note the kink in the shape close to $x=-0.5$.

waveguide in figure 8 have disappeared, indicating that only small reflections occur in the optimal horn.

The optimal designs obtained without smoothing are presented in figure 9 (a). In the vicinity of the ends of the design boundary they show peculiar kinks, enlarged close to $x=-0.5$ in figure 10 . Those kinks are probably due to a large sensitivity in $\nabla_{\alpha} J$ in those regions during the first iterations, causing local deflections and trapping of the optimization into a local minimum.

The reflection spectra for the optimal designs obtained without smoothing are presented in figure 11(a), showing small reflections for the three frequencies. It should be pointed out that non-uniqueness of solutions to the shape optimization problem is to be expected in the single frequency case. Since the number of degrees of freedom is large, the exclusion of one of these can often be compensated by the others. If a shape is found such that $J=0$, we can expect the effect of a small change in one of the design parameters to be counteracted by changes in the other design parameters.

In table 3, data from the optimization for $550 \mathrm{~Hz}$ with and without smoothing are presented. The optimization method converges in less than 20 iterations to a design providing an objective function that is almost five orders of magnitude smaller than the initial $J$. If the optimization is performed without smoothing, convergence is obtained in six iterations.

Furthermore, the norm of the gradient decreases with almost eight orders of magnitude if smoothing is employed and almost eleven orders of magnitude if it is not. However, the norms are different in the two cases. To be able to compare, the last column of table 3 shows the $\|\cdot\|_{0}$-norm (defined as in expression (27)) of the gradient at the optimum. The gradients at optimum are very small, about $10^{-11}$, indicating a well converged result.

In figure 13(a) and 13(b), the behavior of $J$ and $\|\nabla J\|$ for single frequency optimization with smoothing is shown as a function of the number of iterations. The behavior of $\|\nabla J\|$ over the last four iterations indicates superlinear convergence close to the optimum. 


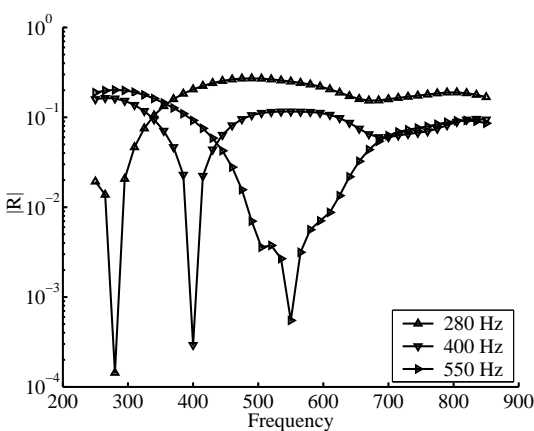

(a) Without smoothing

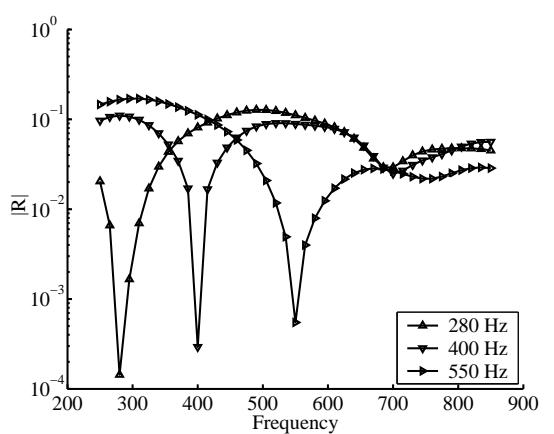

(b) With smoothing

FiguRE 11: Reflection spectra for three horns, each optimized for one frequency, with and without smoothing. The frequencies are $280 \mathrm{~Hz}, 400 \mathrm{~Hz}$, and $550 \mathrm{~Hz}$.

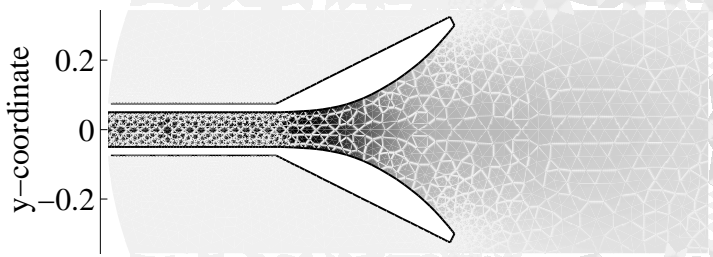

FiguRE 12: The square of the absolute value of the sound pressure distribution at $550 \mathrm{~Hz}$ after optimization. Note that the banded structure in the waveguide shown in figure 8 has disappeared.

TABLE 3: Data from an optimization with smoothing and one without smoothing at $400 \mathrm{~Hz}$.

\begin{tabular}{|c|c|c|c|c|c|}
\hline Method & Iter & Time & $\log \left(\frac{J_{\text {init }}}{J_{\text {opt }}}\right)$ & $\log \left(\frac{\left\|J_{\text {init }}\right\|}{\left\|\nabla J_{\text {opt }}\right\|}\right)$ & $\left\|\nabla J_{\text {opt }}\right\|_{0}$ \\
\hline \hline Smoothing & 19 & $\sim 1 \mathrm{~min}$ & 4.77 & 7.72 & $1.83 \mathrm{e}-11$ \\
No smoothing & 6 & $\sim 1 / 2 \mathrm{~min}$ & 4.77 & 10.7 & $6.03 \mathrm{e}-13$ \\
\hline
\end{tabular}




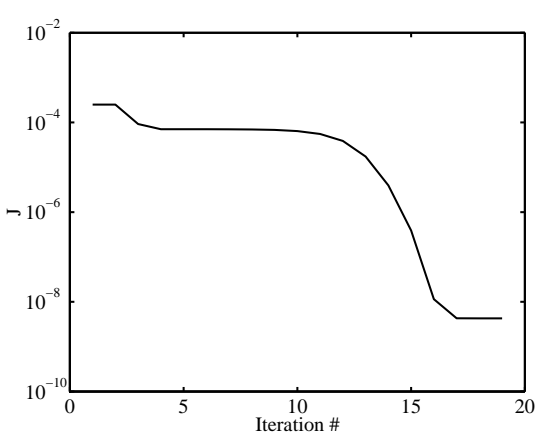

(a) $J$

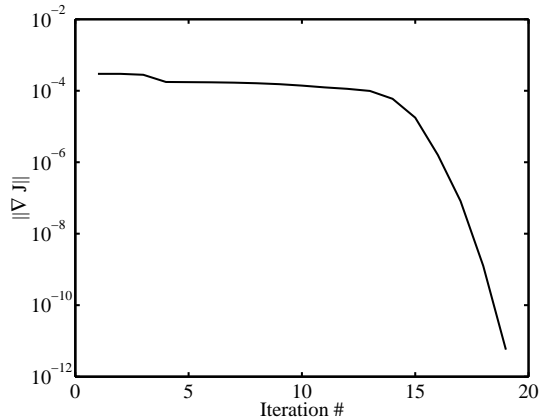

(b) $\|\nabla J\|$

FIGURE 13: The objective function value and the norm of the gradient as a function of iteration number. Smoothing is used.

\subsection{Multi frequency optimization}

The experiments presented above show that the optimization method works well for a single frequency, and the resulting design carries hardly any reflections at the design frequency. However, away from the design frequency, the reflections are much larger. For a loudspeaker, this is not a desired property. It is certainly to be preferred that reflections are kept small within a range of frequencies.

To find an optimal design for a number of frequencies simultaneously, the objective function has to be redefined to take the contribution from the different frequencies into account. Denoting the objective function defined in (10) $J\left(\alpha ; \omega_{j}\right)$ to emphasize that it depends on the frequency $\omega_{j}$, the objective function for multi frequency optimization is defined as

$$
\mathcal{J}^{\epsilon}(\eta, \boldsymbol{\omega})=\frac{\epsilon}{2} \int_{\Gamma_{d}^{r e f}} \eta^{2} d \Gamma+\sum_{j=1}^{M} J\left(\alpha ; \omega_{j}\right),
$$

where $\boldsymbol{\omega}=\left[\omega_{1}, \ldots, \omega_{M}\right]$ is a vector containing the $M$ considered frequencies. $\mathcal{J}^{\epsilon}(\eta, \boldsymbol{\omega})$ will henceforth be referred to as $J$. The gradient of $J$ follows naturally from the definition as the sum of the $M$ single frequency gradients $\nabla J\left(\alpha ; \omega_{j}\right)$, plus the gradient of the regularization term. (Note however that all numerical experiments reported here were performed with $\epsilon=0$.)

\subsubsection{Two frequencies}

Optimization with and without smoothing was performed for the two frequencies 350 and $450 \mathrm{~Hz}$. The resulting designs are shown in figure 14. Note the sharp kink close to $x=-0.5$ in the design obtained when smoothing is not used. The corresponding reflection spectra are presented in figure 15. The horn shape obtained when smoothing is used provides a smaller reflection, in almost the entire frequency band, than the horn obtained without smoothing.

The data in table 4 assures that an unconverged solution is not the cause of the irregular shape obtained when not using smoothing. The solutions are 


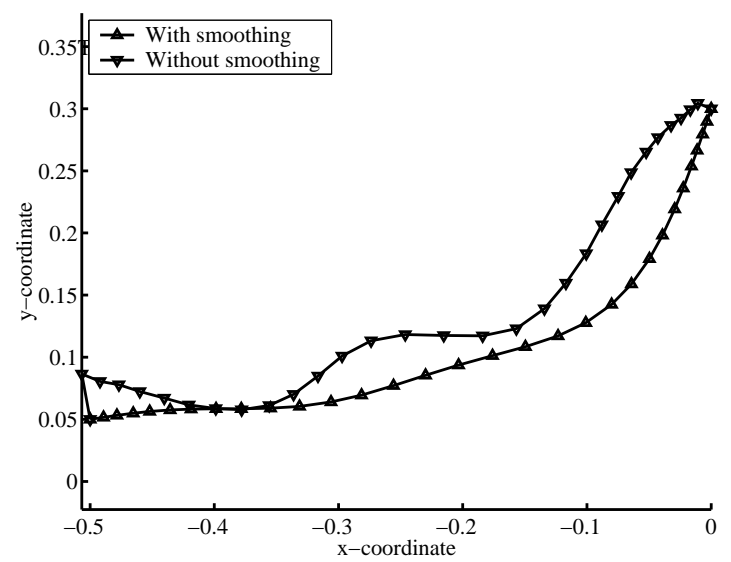

Figure 14: $\Gamma_{d}$ optimized for the two frequencies 350 and $450 \mathrm{~Hz}$. Note the kink at $x=-0.5$ when smoothing is not used.

TABle 4: Data from a multi-point optimization with smoothing and one without smoothing at 350 and $400 \mathrm{~Hz}$.

\begin{tabular}{|c|c|c|c|c|c|}
\hline Method & Iter & Time & $\log \left(\frac{J_{\text {init }}}{J_{\text {opt }}}\right)$ & $\log \left(\frac{\left\|\nabla J_{\text {init }}\right\|}{\| \nabla \text { oppt }_{\|} \|}\right)$ & $\left\|\nabla J_{\text {opt }}\right\|_{0}$ \\
\hline \hline Smoothing & 50 & $\sim 5 \mathrm{~min}$ & 5.03 & 7.64 & $4.56 \mathrm{e}-11$ \\
No smoothing & 19 & $\sim 2 \mathrm{~min}$ & 5.03 & 12.7 & $1.00 \mathrm{e}-14$ \\
\hline
\end{tabular}

well converged, both when smoothing is used and when it is not. Note that the number of iterations has increased with roughly a factor of three compared to the single frequency case.

\subsubsection{Three frequencies}

If optimization for three frequencies without smoothing is attempted, the mesh is destroyed by the oscillating design. In figure 16 the initial design is shown together with an oscillating design provided by optimization without smoothing. Note that this is not a converged solution to the optimization problem.

Figure 17 shows the corresponding finite-element mesh in the vicinity of $x=0$. Due to the "spikes" in the design, the element close to the point $(0,0.3)$ is wrenched in such a way that it ends up on the outside of the boundary. This mesh corruption leads to non-physical solutions to the Helmholtz equation and a break-down of the optimization. Optimization with smoothing works well, however.

\subsubsection{Many frequencies}

Optimization with smoothing for 27 frequencies, equally distributed in the range 310 to $700 \mathrm{~Hz}$, was performed on mesh III. The use of smoothing was necessary in order to avoid mesh corruption. The resulting optimal design is shown in figure 18, and a three dimensional depiction of the horn is shown in figure 19. The reflection spectrum corresponding to the horn in figure 18 is shown in 


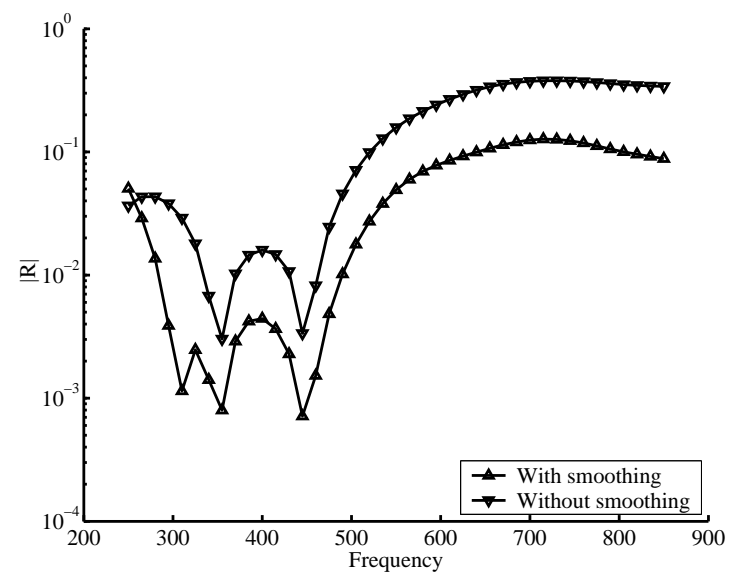

FiguRE 15: Reflection spectra for the solution obtained with and without smoothing. The shape is optimized for 350 and $450 \mathrm{~Hz}$.

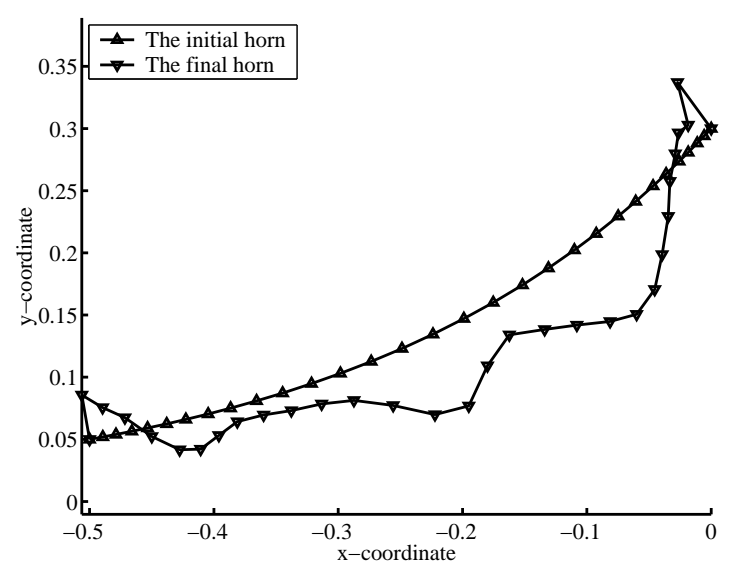

FiguRE 16: The design obtained when the optimization stalled for the three frequencies 350,450 , and $550 \mathrm{~Hz}$. The initial and the final shape are presented. Note that the solution is not converged. 


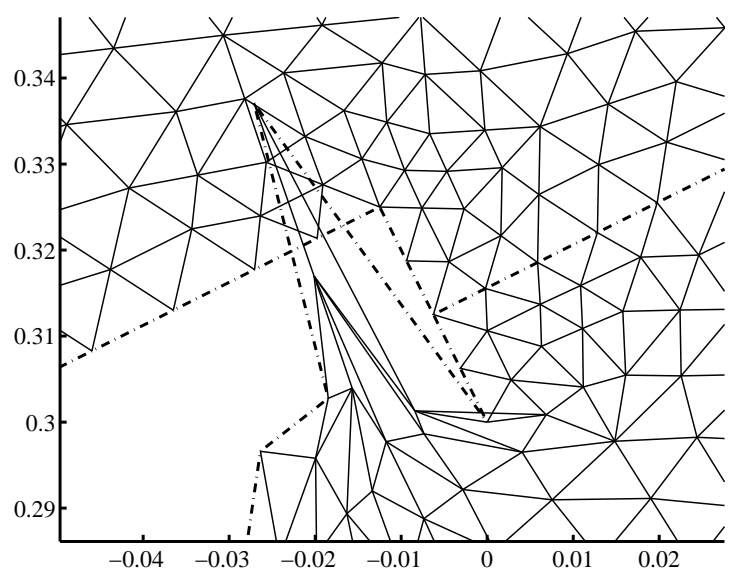

Figure 17: A detail of the design boundary and the mesh corresponding to the final shape obtained when not using smoothing for the three frequencies 350 , 450 and $550 \mathrm{~Hz}$. Note the flat element close to the point $(0,0.3)$ that has been wrenched inside-out by the optimization. Initially it belonged to the interior of $\Omega_{h}$.

TABLE 5: Data from a multi frequency optimization with smoothing in the frequency range between $310 \mathrm{~Hz}$ and $700 \mathrm{~Hz}$.

\begin{tabular}{|c|c|c|c|c|c|}
\hline Method & Mesh & Time & Iter & $\log \left(\frac{J_{\text {init }}}{J_{\text {opt }}}\right)$ & $\log \left(\frac{\left\|\nabla J_{\text {init }}\right\|}{\left\|\nabla J_{\text {opt }}\right\|}\right)$ \\
\hline \hline Smoothing & III & $\sim 16 h$ & 92 & 2.81 & 4.06 \\
\hline
\end{tabular}

figure 20. The amplitude of the reflected wave is smaller than $0.5 \%$ of the amplitude of the incident wave in the entire frequency range between 310 and $700 \mathrm{~Hz}$

Data from this multi-point optimization are presented in table 5. Convergence was achieved in 92 iterations and the value of $J$ decreased with almost three orders of magnitude and the norm of $\nabla J$ with about four orders of magnitude, computed in $\|\cdot\|_{2}$-norm. The behavior of the objective function and the norm of its gradient are shown in figure 21. The convergence rate was not as high as in the single or two frequency case, but a shape was found for which the reflection coefficient exhibits a low and even level throughout the design frequency band.

The slightly wavy shape of the horn in figure 18 is intriguing. To investigate the significance of the wavy shape, a simple sensitivity analysis was performed where the obtained optimal shape was approximated with an interpolating spline. As control points of the spline, the end points of $\Gamma_{d}^{r e f}$ and the three "humps" in the optimal shape were chosen. The result is the very smooth shape shown in figure 22. However, the corresponding reflection spectrum, shown in figure 23 together with the reflection spectra corresponding to the initial and optimal designs, shows that this design is suboptimal for frequencies in the range between 290 and $720 \mathrm{~Hz}$. 


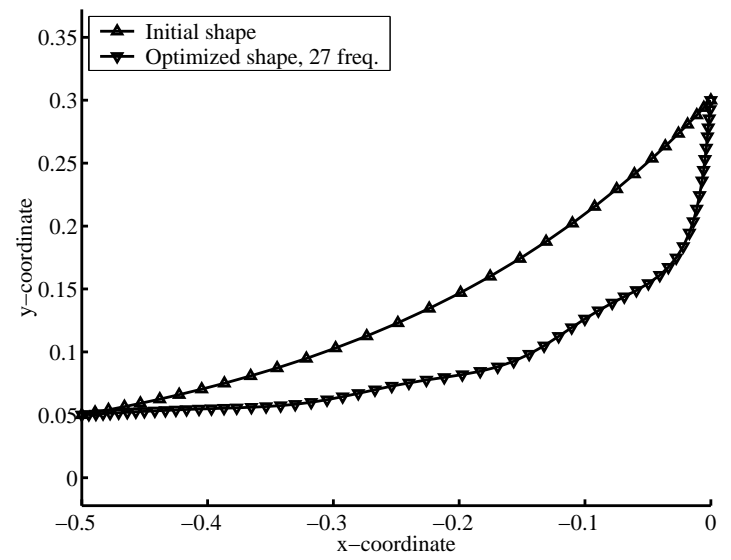

Figure 18: Optimal $\Gamma_{d}$ for 27 frequencies in the frequency band between 310 and $705 \mathrm{~Hz}$.

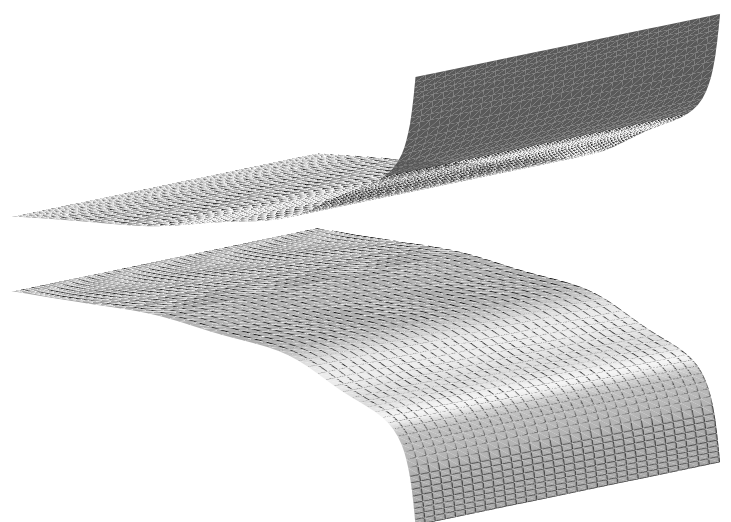

Figure 19: The horn optimized for 27 frequencies between 310 and $700 \mathrm{~Hz}$. 


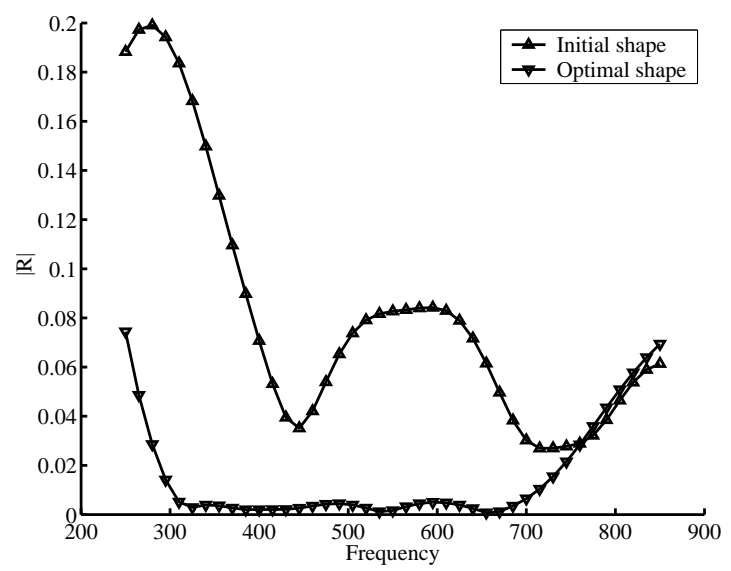

FiguRE 20: The reflection spectrum for the horn optimized for 27 frequencies in the frequency band $310-700 \mathrm{~Hz}$

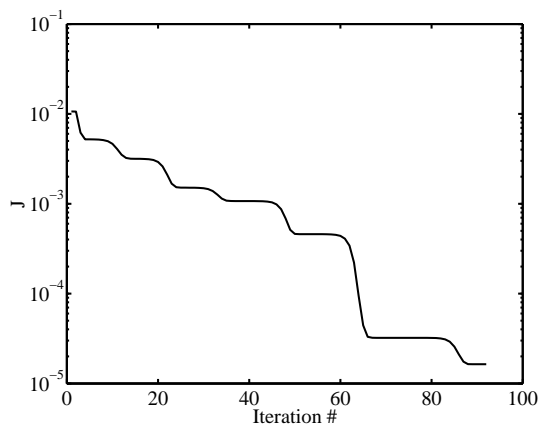

(a) $J$

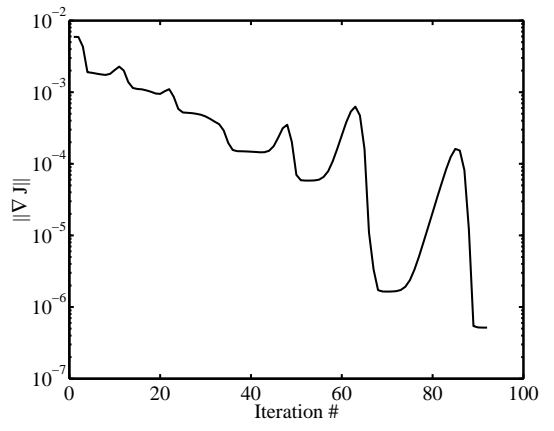

(b) $\|\nabla J\|$

FIGURE 21: The objective function value and the norm of the gradient as a function of iteration number for the 27 -frequency case. 


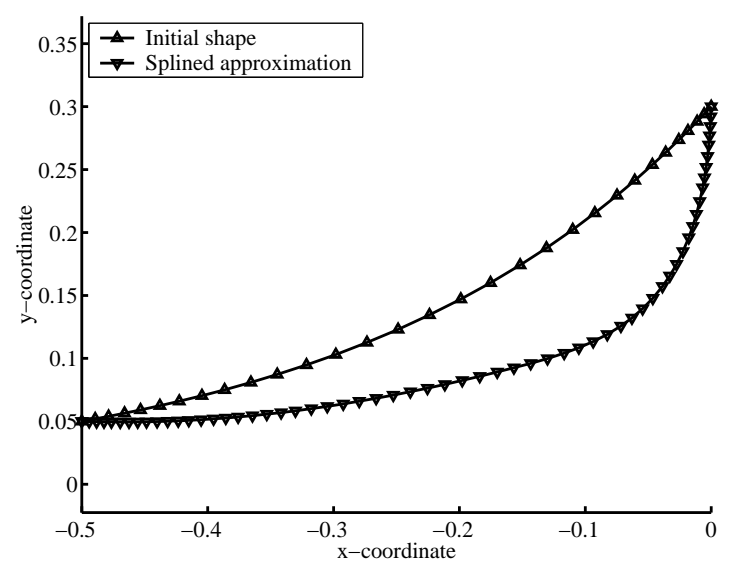

Figure 22: The initial shape and the splined approximation of the optimal shape from the 27 frequency optimization shown in figure 18.

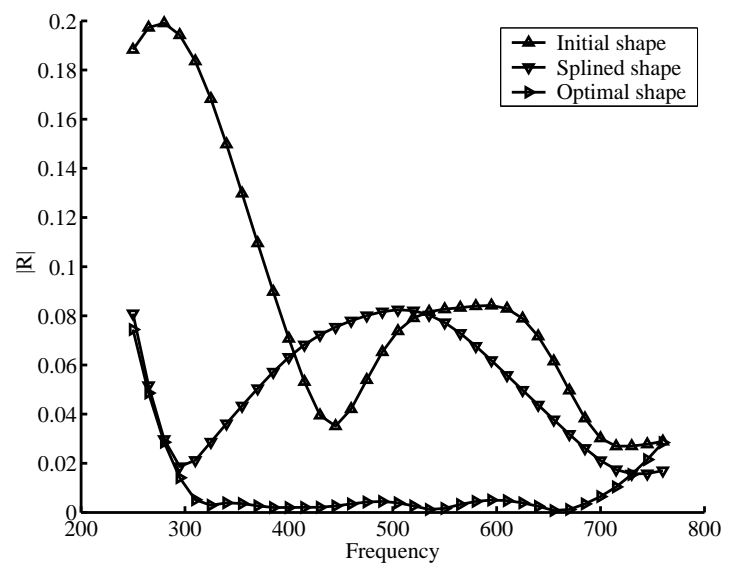

FIGURE 23: The reflection spectra for the initial shape, the optimized shape for 27 frequencies, and the splined approximation. 


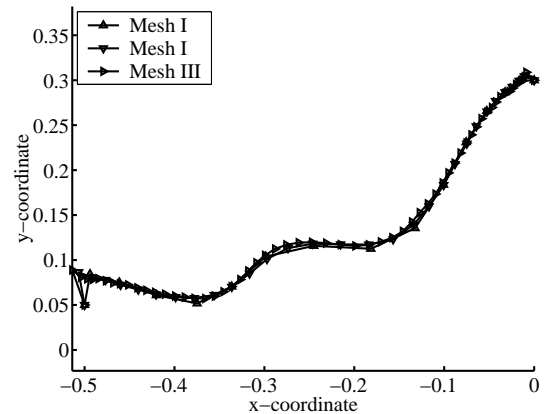

(a) The control boundary

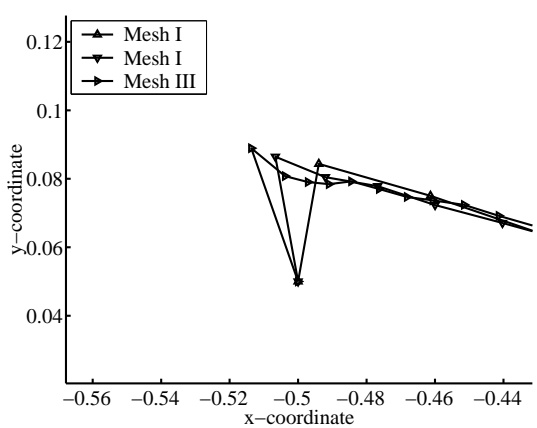

(b) Detail

Figure 24: A optimal design boundary, and a detail close to $x=-0.5$ enlarged, computed for Mesh I, II, III plotted together. The optimization is performed for 350 and $450 \mathrm{~Hz}$ simultaneously and the solutions are well converged.

\subsection{Mesh dependency}

An important question is whether we adequately resolved the problem. If not, the solution to the optimization problem will be distinctly mesh dependent. The results presented above when smoothing is not used are suspicious enough to grant a mesh dependency study. We consider a two-frequency optimization for frequencies 350 and $450 \mathrm{~Hz}$ performed on meshes I, II, and III. The final designs in Figure 24 show a fairly strong mesh dependency. The finer the mesh, the sharper and longer the "spike" at $x=-0.5$, enlarged in figure $24(\mathrm{~b})$, becomes. There is also a smaller "spike" appearing at $x=0$. These results suggest that the optimization algorithm catches a local minimum to the discrete problem that does not converge to something meaningful as the discretization is refined. Note that the reference geometry has "reentrance corners" at $x=-0.5$ and $x=0$, that is, the domain has wedges with angles greater than $180^{\circ}$ at these points. Associated with reentrance corners, there is typically a loss of regularity in solutions to elliptic problems $[15, \S 4.5]$. The gradient expression (20) contains derivatives of the state and adjoint equations evaluated at the design boundary, and these may contain singularities, caused by the reentrant corners, at the end points. This would lead to a high sensitivity of the objective function to local changes at the end points, which could cause effects like the ones seen in Figure 24.

In contrast, the results presented above when smoothing is used appear innocuous. However, to rule out the presence of unresolved irregularities hidden by the smoothing, it would be reassuring to check that no mesh-dependent glitches in the optimal designs appear when the mesh is refined. To check this, we solve a 19-frequency optimization problem on meshes II and III. The frequencies are chosen in $15 \mathrm{~Hz}$ intervals between 305 and $575 \mathrm{~Hz}$. The consistency between the optimal designs shown in figure 25 (a) is very good and this is emphasized by the plot of the relative difference between the two designs shown in figure 25(b). This result leads us to believe that there is indeed a smooth solution to optimization problem (12), even without actively imposing bounds on oscillations 


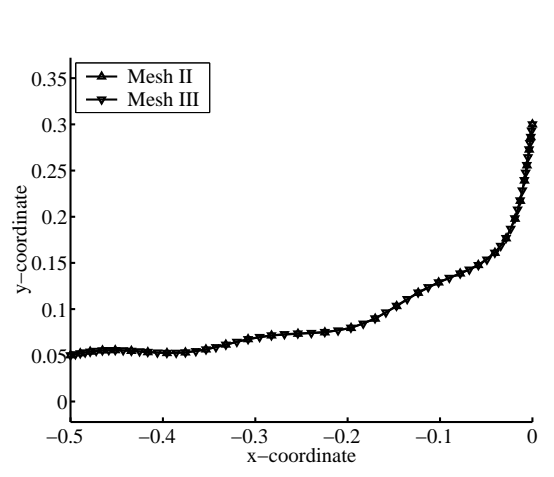

(a) Coinciding designs

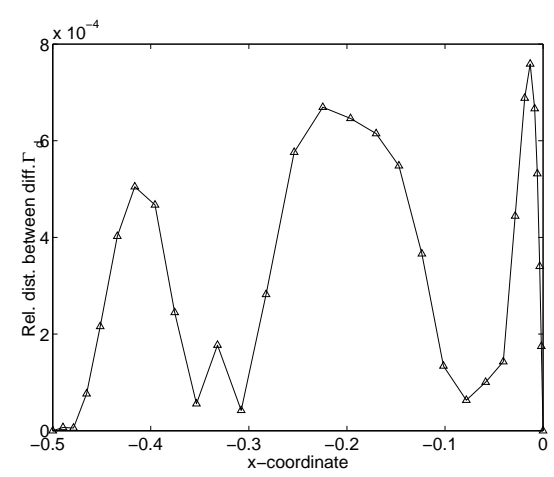

(b) Relative difference

FIGURE 25: The two nearly coinciding designs obtained from optimization performed on Mesh II and III and the relative difference between the two designs.

in $\alpha$. However, the use of smoothing seems necessary to find this solution, otherwise the algorithm may be trapped in local minima associated with wiggly shapes. 


\section{Conclusions}

The work presented in this report shows one approach to the problem of optimizing the shape of an acoustic horn. The method is tailored towards a large number of design variables. For such cases, the method is at the same time relatively inexpensive and robust with respect to its inherent property of avoiding intermediate wiggly iterates and non-smooth designs associated to local minima of the objective function. This feature is accompished through the choice of an appropriate norm. Smooth shapes are favorable both with respect to computational issues, mainly the task of grid generation, and with respect to purely practical questions regarding the manufacturability of a proposed design. Although the search method has a preference to search for smooth solutions, we do not reduce the size of the search space as would have been the case had we imposed constraints on the design parameter space. At first glance, it might appear that the use of smoothing is in effect equivalent to imposing constraints on the parameter space. This is not the case, however, since the size of the search space is in fact kept intact. Any minimum of $J$ is in principle possible to find, although convergence may be slow for minima corresponding to irregular shapes.

The results from the experiments in section 5.3.3 show a shape that, by and large, looks like a conventional loudspeaker horn, but the slightly wavy shape of the horn profile is nonintuitive. The sensitivity analysis performed shows that these oscillations indeed are necessary for the function of the horn. It is a well known fact from horn theory, consistent with our observations, that the impedance and reflection properties of acoustic horns are quite sensitive to geometry changes.

There are no conceptual difficulties that prevent an extension of the presented method to three dimensions. However, details of such a generalization would have to be worked out. In particular, the whole apparatus concerning the design boundary and its modifications would become more complicated. To explicitly compute the matrix $\mathbf{A}$, as described in section 4.3, may become exceedingly expensive. It may be advantageous to instead compute the action of the matrix by solving the discrete counterpart to equation (43), and its adjoint, in each iteration.

Note, however, that a generalization of the geometry to three dimensions does not necessarily mean that the design boundary would have to be extended to a general two-dimensional surface. A number of one-dimensional design curves might still be used, such as would be the case in the design of a horn loudspeaker with rectangular cross section along the horn axis. The cost of evaluating the objective function for a three-dimensional geometry is on the other hand high, irrespective of the boundary representation. 


\section{Acknowledgments}

The authors would like to thank Leif Abrahamsson and Douglas Arnold for discussions and valuable input during the course of this work. Moreover, thanks to Henrik Ekström and Linda Brus for their contributions to the mesh movement algorithm. 


\section{References}

[1] N. Amir, V. Pagneux, and J. Kergomard. Wave propagation in acoustic horns through modal decomposition. Proceedings of the Institute of Acoustics, 19:473-478, 1997.

[2] N. Amir, G. Rosenhouse, and U. Shimony. A discrete model for tubular acoustic systems with varying cross section - the direct and inverse problems. Parts 1 and 2: Theory and experiment. Acustica, 81:450-474, 1995.

[3] A. H. Benade and E. V. Jansson. On plane and spherical waves in horns with nonuniform flare. I. Theory of radiation, resonance frequencies, and mode conversion. Acustica, 31:79-98, 1974.

[4] R. Caussé, J. Kergomard, and X. Lurton. Input impedance of brass musical instruments - comparison between experiment and numerical models. $J$. Acoust. Soc. Am., 75:241-254, 1984.

[5] S. Christensen and N. Olhoff. Shape optimization of a loudspeaker diaphragm with respect to sound directivity properties. Control and Cybernetics, 27(2):177-198, 1998.

[6] R. Dautray and J. L. Lions. Mathematical Analysis and Numerical Methods for Science and Technology, volume 2: Functional and Variational Methods. Springer, Berlin, 1988.

[7] J. E. Dennis and R. B. Schnabel. Numerical Methods for Unconstrained Optimization and Non-linear Equations. Prentice-Hall, 1983.

[8] B. Engquist and A. Majda. Absorbing boundary conditions for the numerical simulation of waves. Mathematics of Computation, 31(139):629-651, 1977.

[9] R.-F. Fehlmann. Computer Simulations and Application of Numerical Techniques for Acoustic Waves in Curved Swiss Horn. PhD thesis, Norwegian Institute of Technology, Department of Telecommunications, Trondheim, 1994.

[10] M. D. Gunzburger, H. Kim, and S. Manservisi. On a shape control problem for the stationary Navier-Stokes equations. Mathematical Modelling and Numerical Analysis, 34(6):1233-1258, 2000.

[11] A. Habbal. Nonsmooth shape optimization applied to linear acoustics. SIAM Journal of Optimization, 8(4):989-1006, Nov. 1998.

[12] A. Jameson. Optimum aerodynamic design using control theory. Computational Fluid Dynamics Review, pages 495-528, 1995.

[13] A. Jameson, J. J. Alonso, J. Reuther, L. Martinelli, and J. C. Vassberg. Aerodynamic shape optimization techniques based on control theory. AIAA Paper 98-2538, 1998.

[14] T. F. Johansen. On the directivity of horn loudspeakers. J. Audio Engineering Soc., 42:1008-1019, 1994. 
[15] C. Johnson. Numerical solution of partial differential equations by the finite element method. Cambridge University Press, 1987.

[16] W. Kausel. Bore reconstruction from measured acoustical input impedance; equipment, signal processing, algorithms and prerequisites. Proceedings of the International Symposium on Musical Acoustics, 2:373-378, 2001.

[17] H.-B. Lee, H.-K. Jung, S.-Y. Hahn, C. Cheon, and K.-S. Lee. Shape optimization of H-plane waveguide tee junction using edge finite element method. IEEE Transactions on Magnetics, 31(3):1928-1931, May 1995.

[18] B. Mohammadi and O. Pironneau. Applied Shape Optimization for Fluids. Oxford University Press, 2001.

[19] Y. Papalambros and D. J. Wilde. Principles of Optimal Design. Cambridge University Press, 2000.

[20] O. Pironneau. Optimal Shape Design for Elliptic Systems. Springer Verlag, 1984.

[21] S. Ratnajeevan, H. Hoole, and S. Sirikumaran. Reflections off aircraft and the shape optimization of a ridged wave guide. IEEE Transactions on Magnetics, 27(5):4150-4153, Sep. 1991.

[22] J. Sokolowski and J.-P. Zolesio. Introduction to Shape Optimization, Shape Sensitivity Analysis. Springer-Verlag, 1992.

[23] A. N. Tikhonov and V. Y. Arsenin. Solutions to Ill-posed Problems. Winston-Wiley, New York, 1977. 


\section{A Gradient derivation}

\section{A.1 Preliminaries}

For the derivation of gradient expression (20), it is convenient to work with the state equation (8) in variational form:

Find $p \in H^{1}(\Omega)$ such that

$$
\begin{aligned}
& c^{2} \int_{\Omega} \nabla \bar{v} \cdot \nabla p d \Omega-\omega^{2} \int_{\Omega} \bar{v} p d \Omega+i \omega c \int_{\Gamma_{\text {in }} \cup \Gamma_{\text {out }}} \bar{v} p d \Gamma+\frac{c^{2}}{2 R_{\Omega}} \int_{\Gamma_{\text {out }}} \bar{v} p d \Gamma \\
&=2 i \omega A \int_{\Gamma_{\text {in }}} \bar{v} d \Gamma \quad \forall v \in H^{1}(\Omega),
\end{aligned}
$$

where $H^{1}(\Omega)$ is the space of complex-valued functions in which the functions as well as their gradients are square-integrable. An overbar denotes complex conjugate. (The variational form above is also the starting point for the finiteelement discretization.)

In order to proceed, we will need to differentiate functions and integrals with respect to domain variations. We will shortly recall definitions and some basic formulas for this. For a more comprehensive treatment, we refer to the book by Sokolowski and Zolesio [22].

Consider a current design $\Gamma_{d}$. Later, we will only consider those designs associated with normal deflections from a reference configuration by formula (11). However, for now, $\Gamma_{d}$ may simply be any smooth shape of the horn. Denote by $\Omega$ the computational domain associated with the current design, and let $p$ be the corresponding solution to the state equation (A.1). A design variation $\delta \varphi: \Gamma_{d} \rightarrow \mathbb{R}^{2}$ is an arbitrary smooth vector field defined on $\Gamma_{d}$ that vanishes at the end points. (Later, we will restrict the design variations to the ones that are compatible with designs given by formula (11), but for the present section it is more convenient to use this more general form.)

Denote by $\Gamma_{d}^{s}$ the current design $\Gamma_{d}$ deflected by the amount $s \delta \varphi$, where $s$ is a small real number; that is, $\boldsymbol{x}_{s} \in \Gamma_{d}^{s}$ if

$$
\boldsymbol{x}_{s}=\boldsymbol{x}+s \delta \boldsymbol{\varphi}(\boldsymbol{x})
$$

for some $\boldsymbol{x} \in \Gamma_{d}$ and for $s \in(-\epsilon, \epsilon)$. The parameter $\epsilon>0$ is chosen sufficiently small so that, for each $s \in(-\epsilon, \epsilon)$, the mapping $\boldsymbol{x} \mapsto \boldsymbol{x}_{s}$ is one-to-one and so that $\Gamma_{d}^{s}$ is smooth and not intersecting any other part of the boundary. Denote by $\Omega_{s}$ the computational domain associated with the deflected design $\Gamma_{d}^{s}$, and denote by $p_{s}$ the solution to equation (A.1) with $\Omega$ replaced by $\Omega_{s}$; note that $p_{0}=p$.

We define the shape derivative (or differential) of $p$ with respect to the design variation $\delta \boldsymbol{\varphi}$ as, for each $\boldsymbol{x} \in \Omega$,

$$
\delta p(\boldsymbol{x})=\lim _{s \rightarrow 0} \frac{p_{s}(\boldsymbol{x})-p(\boldsymbol{x})}{s}=\left.\frac{d}{d x} p_{s}(\boldsymbol{x})\right|_{s=0}
$$

provided that the limit exists. We may also allow the test functions $v$ in equation (A.1) to vary smoothly with $s$ and define $\delta v$ similarly as in expression (A.3).

Let $h_{s}$ be any function, such as $p_{s}$, defined on $\Omega_{s}$ and varying smoothly with $s$. Differentiation of domain integrals with respect to $\delta \varphi$ is defined by

$$
\delta\left(\int_{\Omega} h d \Omega\right)=\lim _{s \rightarrow 0} \frac{1}{s}\left(\int_{\Omega_{s}} h_{s} d \Omega-\int_{\Omega} h d \Omega\right) .
$$


Differentiation of boundary integrals are defined similarly.

Lemma A.1 provides a basic formula that are needed in the derivations. For a proof, we refer to Sokolowski and Zolesio [22], chapter 2.31.

Lemma A.1.

$$
\delta\left(\int_{\Omega} h d \Omega\right)=\int_{\Omega} \delta h d \Omega+\int_{\Gamma_{d}} \boldsymbol{n} \cdot \delta \boldsymbol{\varphi} h d \Gamma
$$

Remark A.1. This is a formula of the type

$$
\delta\left(\int_{\Omega} f d \Omega\right)=\int_{\Omega} \delta f d \Omega+\int_{\delta \Omega} f d \Omega,
$$

that is, a product rule for the differentiation of integrals.

\section{A.2 Differentiation of $J$}

Considering objective function (13) evaluated for a $p$ associated with the current design $\Gamma_{d}$. Differentiating the objective function with respect to the design variation $\delta \varphi$, we obtain

$$
\delta J=\frac{1}{2} \int_{\Gamma_{\text {in }}}[\overline{\delta p}(p-A)+(\bar{p}-\bar{A}) \delta p] d \Gamma=\mathfrak{R e} \int_{\Gamma_{\text {in }}} \overline{\delta p}(p-A) d \Gamma .
$$

Note that only the integrand needs to be differentiated since $\Gamma_{i n}$ does not depend on the design variation.

In order to obtain an expression for how $\delta p$ depends on $\delta \boldsymbol{\varphi}$, we will differentiate state equation (A.1). Let $v \in H^{1}(\Omega)$ be arbitrary. When differentiating the first two terms of expression (A.1) - the terms involving domain integrals - we utilize Lemma A.1 and the product rule for the integrand. Since $\Gamma_{i n}$ and $\Gamma_{\text {out }}$ do not depend on the design variation, only the integrands have to be differentiated for the remaining terms in equation (A.1). Together this yields that

$$
\begin{aligned}
0 & =c^{2} \int_{\Omega} \nabla \delta \bar{v} \cdot \nabla p d \Omega+c^{2} \int_{\Omega} \nabla \bar{v} \cdot \nabla \delta p d \Omega+c^{2} \int_{\Gamma_{d}} \boldsymbol{n} \cdot \delta \boldsymbol{\varphi} \nabla \bar{v} \cdot \nabla p d \Gamma \\
& -\omega^{2} \int_{\Omega} \delta \bar{v} p d \Omega-\omega^{2} \int_{\Omega} \bar{v} \delta p d \Omega-\omega^{2} \int_{\Gamma_{d}} \boldsymbol{n} \cdot \delta \boldsymbol{\varphi} \bar{v} p d \Gamma \\
& +i \omega c \int_{\Gamma_{\text {in }} \cup \Gamma_{\text {out }}} \delta \bar{v} p d \Gamma+i \omega c \int_{\Gamma_{i n} \cup \Gamma_{\text {out }}} \bar{v} \delta p d \Gamma \\
& +\frac{c^{2}}{2 R_{\Omega}} \int_{\Gamma_{\text {out }}} \delta \bar{v} p d \Gamma+\frac{c^{2}}{2 R_{\Omega}} \int_{\Gamma_{\text {out }}} \bar{v} \delta p d \Gamma-2 i \omega c A \int_{\Gamma_{\text {in }}} \delta \bar{v} d \Gamma .
\end{aligned}
$$

Rearranging the terms in expression (A.5) (grouping together the terms involv- 
ing $\delta \bar{v}$ and $\delta p$ ), we find

$$
\begin{aligned}
0=c^{2} & \int_{\Omega} \nabla \delta \bar{v} \cdot \nabla p d \Omega-\omega^{2} \int_{\Omega} \delta \bar{v} p d \Omega+i \omega c \int_{\Gamma_{i n} \cup \Gamma_{\text {out }}} \delta \bar{v} p d \Gamma \\
& +\frac{c^{2}}{2 R_{\Omega}} \int_{\Gamma_{\text {out }}} \delta \bar{v} p d \Gamma-2 i \omega c A \int_{\Gamma_{\text {in }}} \delta \bar{v} d \Gamma \\
+ & c^{2} \int_{\Omega} \nabla \delta p \cdot \nabla \bar{v} d \Omega-\omega^{2} \int_{\Omega} \delta p \bar{v} d \Omega+i \omega c \int_{\Gamma_{\text {in }} \cup \Gamma_{\text {out }}} \delta p \bar{v} d \Gamma \\
& +\frac{c^{2}}{2 R_{\Omega}} \int_{\Gamma_{\text {out }}} \delta p \bar{v} d \Gamma \\
+ & c^{2} \int_{\Gamma_{d}} \boldsymbol{n} \cdot \delta \varphi \nabla \bar{v} \cdot \nabla p d \Gamma-\omega^{2} \int_{\Gamma_{d}} \boldsymbol{n} \cdot \delta \boldsymbol{\varphi} \bar{v} p d \Gamma .
\end{aligned}
$$

The first five terms in expression (A.6) vanish due to state equation (A.1) (with $v=\delta v$ ), leaving us with

$$
\begin{aligned}
0=c^{2} & \int_{\Omega} \nabla \overline{\delta p} \cdot \nabla v d \Omega-\omega^{2} \int_{\Omega} \overline{\delta p} v d \Omega-i \omega c \int_{\Gamma_{i n} \cup \Gamma_{\text {out }}} \overline{\delta p} v d \Gamma \\
& +\frac{c^{2}}{2 R_{\Omega}} \int_{\Gamma_{\text {out }}} \overline{\delta p} v d \Gamma+c^{2} \int_{\Gamma_{d}} \boldsymbol{n} \cdot \delta \boldsymbol{\varphi} \nabla v \cdot \nabla \bar{p} d \Gamma-\omega^{2} \int_{\Gamma_{d}} \boldsymbol{n} \cdot \delta \boldsymbol{\varphi} v \bar{p} d \Gamma
\end{aligned}
$$

after complex conjugation.

Now, let $z \in H^{1}(\Omega)$ be the solution to

$$
\begin{aligned}
c^{2} \int_{\Omega} \nabla \bar{w} \cdot \nabla z d \Omega & -\omega^{2} \int_{\Omega} \bar{w} z d \Omega-i \omega c \int_{\Gamma_{\text {in }} \cup \Gamma_{\text {out }}} \bar{w} z d \Gamma \\
& +\frac{c^{2}}{2 R_{\Omega}} \int_{\Omega} \bar{w} z d \Omega=\int_{\Gamma_{\text {in }}} \bar{w}(p-A) d \Gamma \quad \forall w \in H^{1}(\Omega) .
\end{aligned}
$$

Problem (A.8) is the variational form of the adjoint equation (19).

Choosing $w=\delta p$ in expression (A.8), it becomes

$$
\begin{aligned}
c^{2} \int_{\Omega} \nabla \overline{\delta p} \cdot \nabla z d \Omega-\omega^{2} \int_{\Omega} \overline{\delta p} z d \Omega-i w c \int_{\Gamma_{\text {in }} \cup \Gamma_{\text {out }}} \overline{\delta p} z d \Gamma \\
+\frac{c^{2}}{2 R_{\Omega}} \int_{\Gamma_{\text {out }}} \overline{\delta p} z d \Gamma=\int_{\Gamma_{\text {in }}} \overline{\delta p}(p-A) d \Gamma .
\end{aligned}
$$

In the chain of expressions (A.5)-(A.7), $v \in H^{1}(\Omega)$ was arbitrary. Now make the choice $v=z$, where $z$ is the solution to the adjoint equation (A.8). Since $z$ satisfies expression (A.9), expression (A.7) with $v=z$ reduces to

$$
\begin{aligned}
0=\int_{\Gamma_{i n}} \overline{\delta p}(p-A) d \Gamma+c^{2} \int_{\Gamma_{d}} \boldsymbol{n} \cdot \delta \varphi \nabla z \cdot \nabla \bar{p} d \Gamma & \\
& -\omega^{2} \int_{\Gamma_{d}} \boldsymbol{n} \cdot \delta \boldsymbol{\varphi} z \bar{p} d \Gamma .
\end{aligned}
$$

Expression (A.10) yields the desired relation between $\delta \varphi$ and $\delta p$ in terms of the solution $z$ to the adjoint equation. Substituting relation (A.10) into 
expression (A.4), we obtain

$$
\delta J=\mathfrak{R e}\left[\omega^{2} \int_{\Gamma_{d}} \boldsymbol{n} \cdot \delta \boldsymbol{\varphi} z \bar{p} d \Gamma-c^{2} \int_{\Gamma_{d}} \boldsymbol{n} \cdot \delta \boldsymbol{\varphi} \nabla z \cdot \nabla \bar{p} d \Gamma\right] .
$$

\section{A.3 The gradient $\nabla_{\alpha} J$}

Expression (A.11) provides derivative information for a design variation around a current design $\Gamma_{d}$. This is not quite enough for our purpose. When the admissible designs are given by formula (11), the design varations will be with respect to the fixed reference design $\Gamma_{d}^{r e f}$. The appropriate abstract gradient expression is of the type (15). This expression contains $\delta \alpha$, a function on $\Gamma_{d}^{r e f}$, so we wish to find a representation of expression (15) as an integral over $\Gamma_{d}^{\text {ref }}$ and not $\Gamma_{d}$, as in expression (A.11). This can be done by a change of variables in expression (A.11) in order to obtain an integration over $\Gamma_{d}^{r e f}$. For details regarding change of variables in surface integrals, we again refer to Sokolewski and Zolesio [22, § 2.17].

To provide the expression, we need some additional notation. Let $\boldsymbol{\Phi}_{\alpha}$ : $\Gamma_{d}^{r e f} \rightarrow \Gamma_{d}$ denote the mapping, defined in expression (11), of points on the reference design onto points on the current design. The inverse mapping of $\boldsymbol{\Phi}_{\alpha}$ is denoted $\boldsymbol{\Phi}_{\alpha}^{-1}$. By composing the solution $p$ to state equation (A.1) and the solution $z$ to adjoint equation (A.8) with the mapping $\boldsymbol{\Phi}_{\alpha}$, we define the functions

$$
\begin{aligned}
& p_{\alpha}=p \circ \boldsymbol{\Phi}_{\alpha}, \\
& z_{\alpha}=z \circ \boldsymbol{\Phi}_{\alpha} .
\end{aligned}
$$

That is, for each $\boldsymbol{x} \in \Gamma_{d}^{r e f}$, we define $p_{\alpha}(\boldsymbol{x})=p\left(\boldsymbol{\Phi}_{\alpha}(\boldsymbol{x})\right)$. $z_{\alpha}$ is defined similarly.

When the current designs are provided by formula (11), the design variations are along the (constant) direction $\boldsymbol{n}_{r e f}$. Thus, the design variations are of the type

$$
\delta \boldsymbol{\varphi}=\boldsymbol{n}_{r e f} \delta \alpha \circ \boldsymbol{\Phi}_{\alpha}^{-1},
$$

where $\delta \alpha$ is a difference between two admissible functions, that is, $\delta \alpha=\alpha-\widetilde{\alpha}$, for $\alpha, \widetilde{\alpha} \in U_{\mathrm{ad}}$.

Making a change of variables in expression (A.11), integrating over $\Gamma_{d}^{r e f}$ instead of $\Gamma_{d}$, and utilizing expressions (A.12) and (A.13) yields

$$
\delta J=\mathfrak{R e} \int_{\Gamma_{d}^{r e f}} \delta \alpha\left[\omega^{2} z_{\alpha} \bar{p}_{\alpha}-c^{2} \nabla z_{\alpha} \cdot \nabla \bar{p}_{\alpha}\right] \gamma d \Gamma,
$$

where $\gamma$ is the weighting function resulting from the change of variables given by

$$
\gamma=\left|\nabla \Phi_{\alpha}\right| \boldsymbol{n}_{r e f} \cdot\left(\nabla \boldsymbol{\Phi}_{\alpha}\right)^{-T} \boldsymbol{n}_{r e f},
$$

in which $\nabla \boldsymbol{\Phi}_{\alpha}$ and $\left|\nabla \boldsymbol{\Phi}_{\alpha}\right|$ are the Jacobian and the Jacobian determinant of the mapping $\boldsymbol{\Phi}_{\alpha}$, respectively [22, Prop. 2.47-2.48].

Comparing expression (A.14) with the abstract form (15), we identify the gradient as

$$
\nabla_{\alpha} J=\gamma \mathfrak{R e}\left(\omega^{2} z_{\alpha} \bar{p}_{\alpha}-c^{2} \nabla z_{\alpha} \cdot \nabla \bar{p}_{\alpha}\right)
$$




\section{A.4 Derivation of $\nabla_{\eta} J^{\epsilon}$}

Now consider the objective function

$$
J^{\epsilon}(\eta)=\frac{\epsilon}{2} \int_{\Gamma_{d}^{r e f}} \eta^{2} d \Gamma+\frac{1}{2} \int_{\Gamma_{i n}}|p(\eta)-A|^{2} d \Gamma,
$$

that is, the regularized objective function of $\S 3.1$. From the design variable $\eta$, the function $\alpha$ needed to define the admissible designs by expression (11) is computed by solving the Poisson problem (22). In variational form, equation (22) is: find $\alpha \in H_{0}^{1}\left(\Gamma_{d}^{r e f}\right)$ such that

$$
\int_{\Gamma_{d}^{r e f}} \beta^{\prime} \alpha^{\prime} d \Gamma=\int_{\Gamma_{d}^{r e f}} \beta \eta d \Gamma \quad \forall \beta \in H_{0}^{1}\left(\Gamma_{d}^{r e f}\right) .
$$

Let $\delta \eta$ be a a given design variation, that is, a difference of two design variables: $\delta \eta=\eta-\widetilde{\eta}$ for $\eta, \widetilde{\eta} \in L^{2}\left(\Gamma_{d}^{r e f}\right)$. The gradient of $J^{\epsilon}$ is the linear functional

$$
\delta J^{\epsilon}=\left\langle\nabla_{\eta} J^{\epsilon}, \delta \eta\right\rangle .
$$

Differentiating objective function (A.17) with respect to $\delta \eta$ yields

$$
\delta J^{\epsilon}=\epsilon \int_{\Gamma_{d}^{r e f}} \delta \eta \eta d \Gamma+\mathfrak{R e} \int_{\Gamma_{i n}} \delta \bar{p}(p-A) d \Gamma .
$$

Since the second term in expression (A.20) is equal to the $\delta J$ of expression (A.4), we may use the derivations of $\S$ A.2 and A.3 and insert expressions (A.14) and (A.16) into (A.20) to find that

$$
\delta J^{\epsilon}=\epsilon \int_{\Gamma_{d}^{r e f}} \delta \eta \eta d \Gamma+\int_{\Gamma_{d}^{r e f}} \delta \alpha \nabla_{\alpha} J d \Gamma .
$$

We proceed to find a relation between $\delta \eta$ and $\delta \alpha$., Let $\beta \in H_{0}^{1}\left(\Gamma_{d}^{r e f}\right)$ be arbitrary. Differentiating equation (A.18) with respect to $\delta \eta$ yields

$$
\int_{\Gamma_{d}^{r e f}} \beta^{\prime} \delta \alpha^{\prime} d \Gamma=\int_{\Gamma_{d}^{r e f}} \beta \delta \eta d \Gamma .
$$

Let $r \in H_{0}^{1}\left(\Gamma_{d}^{r e f}\right)$ be the solution to problem

$$
\int_{\Gamma_{d}^{r e f}} w^{\prime} r^{\prime} d \Gamma=\int_{\Gamma_{d}^{r e f}} w \nabla_{\alpha} J d \Gamma . \quad \forall w \in H_{0}^{1}\left(\Gamma_{d}^{r e f}\right),
$$

Equation (A.23) is a variational form of equation (25) and constitutes the adjoint with respect to equation (A.18). Choosing $w=\delta \alpha$ in expression (A.23) and $\beta=r$ in expression (A.22) yields that

$$
\int_{\Gamma_{d}^{r e f}} \delta \alpha^{\prime} r^{\prime} d \Gamma=\int_{\Gamma_{d}^{r e f}} \delta \alpha \nabla_{\alpha} J d \Gamma
$$

and

$$
\int_{\Gamma_{d}^{r e f}} r^{\prime} \delta \alpha^{\prime} d \Gamma=\int_{\Gamma_{d}^{r e f}} r \delta \eta d \Gamma
$$


Combining expressions (A.24) and (A.25), we find the desired relation between $\delta \eta$ and $\delta \alpha$,

$$
\int_{\Gamma_{d}^{r e f}} r \delta \eta d \Gamma=\int_{\Gamma_{d}^{r e f}} \delta \alpha \nabla_{\alpha} J d \Gamma .
$$

Finally substituting expression (A.26) into (A.21) yields

$$
\delta J^{\epsilon}=\epsilon \int_{\Gamma_{d}^{r e f}} \delta \eta \eta d \Gamma+\int_{\Gamma_{d}^{r e f}} \delta \eta r d \Gamma .
$$

Comparing expression (A.27) with the abstract form (A.19), we may identify the gradient as

$$
\nabla_{\eta} J^{\epsilon}=\epsilon \eta+r .
$$




\section{B Gradient derivation in the discrete case}

\section{B.1 Preliminaries}

Consider the discrete state equation (30) and the discrete objective function (32). Our aim is to derive expression (40) giving the gradient with respect to each mesh point in $\Omega_{h} \cup \Gamma_{d}$, the strict interior of the domain and the design boundary. (The final gradient expression with respect to a variation of the design boundary will depend on the mesh movement strategy, as discussed in $\S$ 4.3.) In order to proceed we need formulas for the differentiated finite-element basis functions and integrals.

Let $K$ be the set of nodal indices in the mesh, and let $K_{\Omega_{h} \cup \Gamma_{d}} \subset K$ be the nodes that are located in the strict interior of the domain or on the design boundary. Moreover, let $\boldsymbol{x}_{k}$, for $k \in K_{\Omega_{h} \cup \Gamma_{d}}$, be an arbitrary mesh point. Let $\delta \boldsymbol{x}_{k}$ be a mesh point variation, which simply is an arbitrary vector in $\mathbb{R}^{2}$. Consider now a perturbation of mesh point $\boldsymbol{x}_{k}$ in the direction $\delta \boldsymbol{x}_{k}$ that yields new mesh points $\boldsymbol{x}_{k}^{s}$, that is,

$$
\boldsymbol{x}_{k}^{s}=\boldsymbol{x}_{k}+s \delta \boldsymbol{x}_{k}
$$

where $s \in(-\epsilon, \epsilon)$ with $\epsilon>0$ small enough so that each $\boldsymbol{x}_{k}^{s}$ yields a mesh with the same topology as the unperturbed mesh. Each triangle in the mesh with node $\boldsymbol{x}_{k}$ as one of its vertices will be altered by the above variation. Thus, each finite-element basis function associated with the nearest neighbors of node $k$ depends on the variation $\delta \boldsymbol{x}_{k}$. Let $w_{j}$, for $j \in K$, be any of the finite-element basis functions, and let $w_{j}^{s}$ be the corresponding basis function subject to the displacement (B.1); note that $w_{j}^{s}$ will be different from $w_{j}$ only when $j$ is equal to $k$ or when $j$ represents one of the nearest neighbors to $k$. We may now define the shape derivative of $w_{j}$ similarly as in expression (A.3); for each $\boldsymbol{x} \in \Omega_{h}$,

$$
\delta w_{j}(\boldsymbol{x})=\lim _{s \rightarrow 0} \frac{w_{j}^{s}(\boldsymbol{x})-w_{j}(\boldsymbol{x})}{s}=\left.\frac{d}{d x} w_{j}^{s}(\boldsymbol{x})\right|_{s=0} .
$$

Thus, $\delta w_{j}$ will be nonzero only when $j$ is equal to $k$ or when $j$ represents one of the nearest neighbors to $k$.

We may also differentiate integrals with respect to the variation $\delta \boldsymbol{x}_{k}$. Let $T_{n}$ be any triangle in the mesh, and let $T_{n}^{s}$ be corresponding triangle subject to the displacement (B.1). If $h_{s}$ is any function defined on a triangle $T_{n}$ in the mesh and varying smoothly with $s$, we define

$$
\delta\left(\int_{T_{n}} h d \Omega\right)=\lim _{s \rightarrow 0} \frac{1}{s}\left(\int_{T_{n}^{s}} h_{s} d \Omega-\int_{T_{n}} h d \Omega\right)
$$

The fundamental formulas that are needed in the derivations are collected in the following lemmas.

Lemma B.1. Subject to a variation $\delta \boldsymbol{x}_{k}$ of mesh node $k \in K_{\Omega_{h} \cup \Gamma_{d}}$, we have

$$
\delta w_{j}=-w_{k} \delta \boldsymbol{x}_{k} \cdot \nabla w_{j} \quad \forall j \in K .
$$

Remark B.1. Note that $w_{j} \in V_{h}$ but $\delta w_{j} \notin V_{h}$, where $V_{h}$ is the space of the finite-element functions, that is, the space of continuous functions, linear on each triangle in the mesh. The function $\delta w_{j}$ is piecewise linear but in general discontinuous at some edges due to the presence of $\nabla w_{j}$. 
Lemma B.2. Subject to a variation $\delta \boldsymbol{x}_{k}$ of mesh node $k \in K_{\Omega_{h} \cup \Gamma_{d}}$, we have

$$
\delta\left(\int_{T_{n}} h d \Omega\right)=\int_{T_{n}} \delta h d \Omega+\delta \boldsymbol{x}_{k} \cdot \int_{T_{n}} \nabla\left(h w_{k}\right) d \Omega,
$$

where $T_{n}$ is any triangle in the mesh, and $h$ is a smooth function defined in $T_{n}$ that varies smoothly with $s$.

Remark B.2. Lemma B.2 is only a rephrasing of Lemma A.1 suitable to the needs of the current section.

For proofs of Lemma B.1 and B.2, we refer to Pironneau [20].

\section{B.2 Derivation}

Differentiating the discrete objective function (32) with respect to a variation $\delta \boldsymbol{x}_{k}$ of mesh node $k \in K_{\Omega_{h} \cup \Gamma_{d}}$, we obtain

$$
\delta J_{h}=\frac{1}{2} \int_{\Gamma_{i n}}[\overline{\delta p}(p-A)+(\bar{p}-\bar{A}) \delta p] d \Gamma=\mathfrak{R e} \int_{\Gamma_{i n}} \overline{\delta p}(p-A) d \Gamma
$$

Note that only the integrand needs to be differentiated since $\Gamma_{\text {in }}$ does not depend on the design variation. The differential $\delta J_{h}$ is related to the partial derivative of $J_{h}$ through

$$
\delta J_{h}=\delta \boldsymbol{x}_{k} \cdot \frac{\partial J_{h}}{\partial \boldsymbol{x}_{k}} .
$$

Note that the partial derivative is a two-vector with components in the two coordinate directions.

Remark B.3. To simplify the notation, the subscript $h$ is suppressed from functions in $V_{h}$ for the rest of this section.

For the solution $p$ to state equation (31), we have that $p \in V_{h}$ and it may thus be expanded in the finite-element basis,

$$
p(\boldsymbol{x})=\sum_{j \in K} p_{j} w_{j}(\boldsymbol{x}) .
$$

Differentiating both sides of expression (B.4) with respect to a design variation $\delta \boldsymbol{x}_{k}$ of node $k$ yields

$$
\begin{aligned}
\delta p & =\sum_{j \in K} \delta p_{j} w_{j}+\sum_{j \in K} p_{j} \delta w_{j} \\
& =\sum_{j \in K} \delta p_{j} w_{j}-\sum_{j \in K} p_{j} w_{k} \delta \boldsymbol{x}_{k} \cdot \nabla w_{j} \\
& =\sum_{j \in K} \delta p_{j} w_{j}-\sum_{j \in K} \delta \boldsymbol{x}_{k} \cdot \nabla\left(p_{j} w_{j}\right) w_{k} \\
& =\delta \tilde{p}-w_{k} \delta \boldsymbol{x}_{k} \cdot \nabla p,
\end{aligned}
$$

where we have used Lemma B.1 in the second equality, and where

$$
\delta \tilde{p}=\sum_{j \in K} \delta p_{j} w_{j}
$$


Note that $\delta \tilde{p} \in V_{h}$ but not $\delta p$ because of the presence of the second term in the right-hand side of expression (B.5); this term, and thus $\delta p$ are in general discontinuous at some edges. Since we have chosen $k \in K_{\Omega_{h} \cup \Gamma_{d}}$, the second term in the right-hand side of expression (B.5) vanishes on all boundaries, except $\Gamma_{d}$. Thus $\delta p=\delta \tilde{p}$ on $\Gamma_{\text {in }}$ and expression (B.2) may be written

$$
\delta J_{h}=\mathfrak{R e} \int_{\Gamma_{i n}} \overline{\delta \tilde{p}}(p-A) d \Gamma .
$$

In order to obtain an expression on how $\delta \tilde{p}$ depends on the mesh point variation we will differentiate the state equation. Consider state equation (30), write the integrals as sums over all triangles and differentiate with respect to $\delta \boldsymbol{x}_{k}$, utilizing Lemma B.2,

$$
\begin{aligned}
& 0=\sum_{n=1}^{M}\left\{c^{2} \int_{T_{n}} \nabla \delta w_{j} \cdot \nabla p d \Omega+c^{2} \int_{T_{n}} \nabla w_{j} \cdot \nabla \delta p d \Omega\right. \\
&+c^{2} \delta \boldsymbol{x}_{k} \cdot \int_{T_{n}} \nabla\left(w_{k} \nabla w_{j} \cdot \nabla p\right) d \Omega-\omega^{2} \int_{T_{n}} \delta w_{j} p d \Omega \\
&\left.\quad-\omega^{2} \int_{T_{n}} w_{j} \delta p d \Omega-\omega^{2} \delta \boldsymbol{x}_{k} \cdot \int_{T_{n}} \nabla\left(w_{k} w_{j} p\right) d \Omega\right\} \\
&+i \omega c \int_{\Gamma_{\text {in }} \cup \Gamma_{\text {out }}} w_{j} \delta p d \Gamma+\frac{c^{2}}{2 R_{\Omega}} \int_{\Gamma_{\text {out }}} w_{j} \delta p d \Gamma \quad \forall j \in K .
\end{aligned}
$$

Note that $\left.\delta w_{j}\right|_{\Gamma_{i n}}=0$ since $k$ is not a node on $\Gamma_{i n}$; this fact has been used to reduce the number of boundary integral terms that appear in expression (B.8). The integrals in expression (B.8) containing $\delta w_{j}$ can be rewritten in the following way using Lemma B.1:

$$
\begin{aligned}
\int_{T_{n}} \nabla \delta w_{j} \cdot \nabla p d \Omega & =-\int_{T_{n}} \nabla\left(w_{k} \delta \boldsymbol{x}_{k} \cdot \nabla w_{j}\right) \cdot \nabla p d \Omega \\
& =-\delta \boldsymbol{x}_{k} \cdot \int_{T_{n}} \nabla w_{j} \nabla w_{k} \cdot \nabla p d \Omega \\
\int_{T_{n}} \delta w_{j} p d \Omega & =-\delta \boldsymbol{x}_{k} \cdot \int_{T_{n}} w_{k} p \nabla w_{j} d \Omega .
\end{aligned}
$$

In the first of the expressions (B.9), we use the fact that the gradient of the test function is constant on each triangle; there would be an additional term involving second derivatives of $w_{j}$ for basis functions of higher order. The third integral in expression (B.8) may likewise be written

$$
\delta \boldsymbol{x}_{k} \cdot \int_{T_{n}} \nabla\left(w_{k} \nabla w_{j} \cdot \nabla p\right) d \Omega=\delta \boldsymbol{x}_{k} \cdot \int_{T_{n}} \nabla w_{k}\left(\nabla w_{j} \cdot \nabla p\right) d \Omega,
$$

again using the fact that the gradient of test functions are constant on each triangle.

Inserting the splitting (B.5) into the integrals of expression (B.8) involving $\delta p$ yields

$$
\begin{aligned}
& \int_{T_{n}} \nabla w_{j} \cdot \nabla \delta p d \Omega=\int_{T_{n}} \nabla w_{j} \cdot \nabla \delta \tilde{p} d \Omega-\delta \boldsymbol{x}_{k} \cdot \int_{T_{n}} \nabla p \nabla w_{j} \cdot \nabla w_{k} d \Omega \\
& \int_{T_{n}} w_{j} \delta p d \Omega=\int_{T_{n}} w_{j} \delta \tilde{p} d \Omega-\delta \boldsymbol{x}_{k} \cdot \int_{T_{n}} w_{j} w_{k} \nabla p d \Omega
\end{aligned}
$$


where the fact that the gradient of $p$ is constant on each triangle has been used to obtain the last term in the first of the expressions (B.11).

Substituting expressions (B.9), (B.10), and (B.11) into (B.8) yields

$$
\begin{aligned}
0=\sum_{n=1}^{M}\{ & -c^{2} \delta \boldsymbol{x}_{k} \cdot \int_{T_{n}} \nabla w_{j} \nabla w_{k} \cdot \nabla p d \Omega+c^{2} \int_{T_{n}} \nabla w_{j} \cdot \nabla \delta \tilde{p} d \Omega \\
& -c^{2} \delta \boldsymbol{x}_{k} \cdot \int_{T_{n}} \nabla p \nabla w_{j} \cdot \nabla w_{k} d \Omega \\
& +c^{2} \delta \boldsymbol{x}_{k} \cdot \int_{T_{n}} \nabla w_{k} \nabla w_{j} \cdot \nabla p d \Omega \\
& +\omega^{2} \delta \boldsymbol{x}_{k} \cdot \int_{T_{n}} w_{k} p \nabla w_{j} d \Omega-\omega^{2} \int_{T_{n}} w_{j} \delta \tilde{p} d \Omega \\
& \left.+\omega^{2} \delta \boldsymbol{x}_{k} \cdot \int_{T_{n}} w_{j} w_{k} \nabla p d \Omega-\omega^{2} \delta \boldsymbol{x}_{k} \cdot \int_{T_{n}} \nabla\left(w_{k} w_{j} p\right) d \Omega\right\} \\
+i \omega c & \int_{\Gamma_{\text {in }} \cup \Gamma_{\text {out }}} w_{j} \delta \tilde{p} d \Gamma+\frac{c^{2}}{2 R_{\Omega}} \int_{\Gamma_{\text {out }}} w_{j} \delta \tilde{p} d \Gamma \quad \forall j \in K .
\end{aligned}
$$

For the last two terms, we have used the fact that $\delta p=\delta \tilde{p}$ on $\Gamma_{\text {in }} \cup \Gamma_{\text {out }}$.

Summing up the integrals, reordering terms, and taking the complex conjugate of expression (B.12) yields

$$
\begin{aligned}
0 & =c^{2} \int_{\Omega} \nabla \overline{\delta \tilde{p}} \cdot \nabla w_{j} d \Omega-\omega^{2} \int_{\Omega} \overline{\delta \tilde{p}} w_{j} d \Omega \\
& -i \omega c \int_{\Gamma_{\text {in }} \cup \Gamma_{\text {out }}} \overline{\delta \tilde{p}} w_{j} d \Gamma+\frac{c^{2}}{2 R_{\Omega}} \int_{\Gamma_{\text {out }}} \overline{\delta \tilde{p}} w_{j} d \Gamma \\
& -c^{2} \delta \boldsymbol{x}_{k} \cdot \int_{\Omega} \nabla w_{j} \nabla w_{k} \cdot \nabla \bar{p} d \Omega-c^{2} \delta \boldsymbol{x}_{k} \cdot \int_{T_{n}} \nabla \bar{p} \nabla w_{j} \cdot \nabla w_{k} d \Omega \\
& +c^{2} \delta \boldsymbol{x}_{k} \cdot \int_{\Omega} \nabla w_{k} \nabla w_{j} \cdot \nabla \bar{p} d \Omega-\omega^{2} \delta \boldsymbol{x}_{k} \cdot \int_{\Omega} w_{j} \bar{p} \nabla w_{k} d \Omega \quad \forall j \in K .
\end{aligned}
$$

Let $z \in V_{h}$ be arbitrary. Expanding $z$ in the finite-element basis,

$$
z(\boldsymbol{x})=\sum_{j \in K} z_{j} w_{j}(\boldsymbol{x}),
$$

multiplying expression (B.13) with $z_{j}$, and summing over all $j \in K$, we obtain

$$
\begin{aligned}
0 & =c^{2} \int_{\Omega} \nabla \overline{\delta \tilde{p}} \cdot \nabla z d \Omega-\omega^{2} \int_{\Omega} \overline{\delta \tilde{p}} z d \Omega-i \omega c \int_{\Gamma_{\text {in }} \cup \Gamma_{\text {out }}} \overline{\delta \tilde{p}} z d \Gamma \\
& +\frac{c^{2}}{2 R_{\Omega}} \int_{\Gamma_{\text {out }}} \overline{\delta \tilde{p}} z d \Gamma-c^{2} \delta \boldsymbol{x}_{k} \cdot \int_{\Omega} \nabla z\left(\nabla w_{k} \cdot \nabla \bar{p}\right) d \Omega \\
& -c^{2} \delta \boldsymbol{x}_{k} \cdot \int_{\Omega} \nabla \bar{p}\left(\nabla z \cdot \nabla w_{k}\right) d \Omega+c^{2} \delta \boldsymbol{x}_{k} \cdot \int_{\Omega} \nabla w_{k}(\nabla z \cdot \nabla \bar{p}) d \Omega \\
& -\omega^{2} \delta \boldsymbol{x}_{k} \cdot \int_{\Omega} z \bar{p} \nabla w_{k} d \Omega
\end{aligned}
$$

So far $z$ has been arbitrary. Now we pick the particular $z \in V_{h}$ being the 
solution to the adjoint equation

$$
\begin{aligned}
c^{2} \int_{\Omega} \nabla w_{j} \cdot \nabla z d \Omega-\omega^{2} \int_{\Omega} w_{j} z d \Omega & -i \omega c \int_{\Gamma_{\text {in }} \cup \Gamma_{\text {out }}} w_{j} z d \Gamma+\frac{c^{2}}{2 R_{\Omega}} \int_{\Gamma_{\text {out }}} w_{j} z d \Gamma \\
& =\int_{\Gamma_{\text {in }}} w_{j}(p-A) d \Gamma \quad \forall j \in K \quad \text { (B.16) }
\end{aligned}
$$

Multiplying the adjoint equation (B.16) with $\bar{\delta}_{j}$ (the complex conjugate of the coefficient in expansion (B.6)), and summing over all $j \in K$ results in

$$
\begin{aligned}
c^{2} \int_{\Omega} \nabla \overline{\delta \tilde{p}} \cdot \nabla z d \Omega-\omega^{2} \int_{\Omega} \overline{\delta \tilde{p}} z d \Omega-i w c \int_{\Gamma_{\text {in }} \cup \Gamma_{\text {out }}} \overline{\delta \tilde{p}} z d \Gamma \\
\quad+\frac{c^{2}}{2 R_{\Omega}} \int_{\Gamma_{\text {out }}} \overline{\delta \tilde{p}} z d \Gamma=\int_{\Gamma_{\text {in }}} \overline{\delta \tilde{p}}(p-A) d \Gamma .
\end{aligned}
$$

Substituting expression (B.17) into (B.15), we obtain

$$
\begin{aligned}
0 & =\int_{\Gamma_{i n}} \overline{\delta \tilde{p}}(p-A) d \Gamma-c^{2} \delta \boldsymbol{x}_{k} \cdot \int_{\Omega} \nabla z\left(\nabla w_{k} \cdot \nabla \bar{p}\right) d \Omega \\
& -c^{2} \delta \boldsymbol{x}_{k} \cdot \int_{\Omega} \nabla \bar{p}\left(\nabla z \cdot \nabla w_{k}\right) d \Omega+c^{2} \delta \boldsymbol{x}_{k} \cdot \int_{\Omega} \nabla w_{k}(\nabla z \cdot \nabla \bar{p}) d \Omega \\
& -\omega^{2} \delta \boldsymbol{x}_{k} \cdot \int_{\Omega} z \bar{p} \nabla w_{k} d \Omega
\end{aligned}
$$

Expression (B.18) provides the desired relation between $\delta \tilde{p}$ and the mesh point variation in terms of the solution $z$ to the adjoint equation. Inserting (B.18) into (B.7) yields

$$
\begin{aligned}
\delta J & =\mathfrak{R e}\left\{c^{2} \delta \boldsymbol{x}_{k} \cdot \int_{\Omega} \nabla z\left(\nabla w_{k} \cdot \nabla \bar{p}\right) d \Omega+c^{2} \delta \boldsymbol{x}_{k} \cdot \int_{\Omega} \nabla \bar{p}\left(\nabla z \cdot \nabla w_{k}\right) d \Omega\right. \\
& \left.-c^{2} \delta \boldsymbol{x}_{k} \cdot \int_{\Omega} \nabla w_{k}(\nabla z \cdot \nabla \bar{p}) d \Omega+\omega^{2} \delta \boldsymbol{x}_{k} \cdot \int_{\Omega} z \bar{p} \nabla w_{k} d \Omega\right\}
\end{aligned}
$$

Finally, comparing expression (B.19) with definition (B.3), noting that $\delta \boldsymbol{x}_{k}$ is real, we can identify the gradient as

$$
\begin{aligned}
\frac{\partial J_{h}}{\partial \boldsymbol{x}_{k}}=\mathfrak{R e}\{ & c^{2} \int_{\Omega} \nabla z\left(\nabla w_{k} \cdot \nabla \bar{p}\right) d \Omega+c^{2} \int_{\Omega} \nabla \bar{p}\left(\nabla z \cdot \nabla w_{k}\right) d \Omega \\
& \left.\quad-c^{2} \int_{\Omega} \nabla w_{k}(\nabla z \cdot \nabla \bar{p}) d \Omega+\omega^{2} \int_{\Omega} z \bar{p} \nabla w_{k} d \Omega\right\} \quad \forall k \in K_{\Omega_{h} \cup \Gamma_{d}} .
\end{aligned}
$$

